\title{
Neuroprotection of the Developing Brain by Dexmedetomidine Is Mediated by Attenuating Single Propofol-induced Hippocampal Apoptosis and Synaptic Plasticity Deficits
}

\author{
Wenchong Sun, Jian Wang, Dasheng Cai and Ling Pei* \\ Department of Anesthesiology, the First Affiliated Hospital, China Medical University, Shenyang 110001, China
}

\begin{abstract}
Dexmedetomidine (DEX) has neuroprotective effects and its efficacy was determined in propofol-treated pups. Postnatal day (P) 7 rats were exposed to propofol and DEX to investigate the induced apoptosis-related gene expression. Furthermore, synaptic structural changes at the cellular level were observed by electron microscopy. Induction of hippocampal long-term potentiation (LTP) of P30 rats and long-lasting performance of spatial discrimination at P30 and P60 were evaluated. After a single propofol exposure to P7 rats, DEX pretreatment effectively rescued the profound apoptosis seen in hippocampal neurocytes, and strongly reversed the aberrant expression levels of Bcl2-like 1 (BCL2L1), matrix metallopeptidase 9 (MMP-9) and cleaved caspase 3 (CC3), and sharply enhanced synaptic plasticity. However, there were no significant differences in escape latency or crossing times in a probe test. This was accompanied by no obvious reduction in search strategies among the rat groups. No impairment of long-term learning and memory in P30 or P60 rats was detected when using a single dose propofol treatment during the most vulnerable period of brain development. DEX was shown to ameliorate the rodent developmental neurotoxicity caused by a single neonatal propofol challenge, by altering MMP-9, BCL2L1 and CC3 apoptotic signaling.
\end{abstract}

Key words: Apoptosis, Dexmedetomidine, Propofol, Synaptic plasticity

\section{INTRODUCTION}

In clinics, millions of infants receive general anesthesia/deep sedation for surgery or medical diagnostic examinations annually. This means that younger infants and even toddlers may receive general anesthetics and sedative drugs at some point in early childhood. The US Food and Drug Administration (FDA) has warned that young children should postpone or even avoid repeated or prolonged exposure to general anesthesia until three years of age. However, the proposal would postpone timely treatment and lead to worsening outcomes.

\footnotetext{
Submitted July 12, 2020, Revised October 11,2020, Accepted October 15, 2020

* To whom correspondence should be addressed. TEL: 86-024-83282910, FAX: 86-024-83282910 e-mail:lingpei49@vip.sina.com
}

Many clinicians and parents observed that day-surgery children experienced transient neurobehavior abnormalities even after a single childhood anesthetic exposure [1]. Experimental studies indicated that early anesthesia exerted neurotoxicity to developing neurocytes and synaptogenesis during the highly vulnerable period of brain growth spurts [2-5]. Propofol (2, 6 - diisopropylphenol), one of the most commonly used general anesthetics in neonates, its underlying mechanism during the development of the mammalian brain has not been thoroughly investigated. To date, the association between a single use of propofol and longterm neurodevelopmental sequalae is still uncertain. The risk of general anesthesia to early developmental vulnerability in the pediatric central nervous system (CNS) continues to attract growing interest from researchers.

Dexmedetomidine (DEX, S-4-[1-(2,3-dimethylphenyl) ethyl]-3H-imidazole) is a highly selective $\alpha 2$ adrenoreceptor agonist and has anti-anxiolytic, sedative, and analgesic properties. 


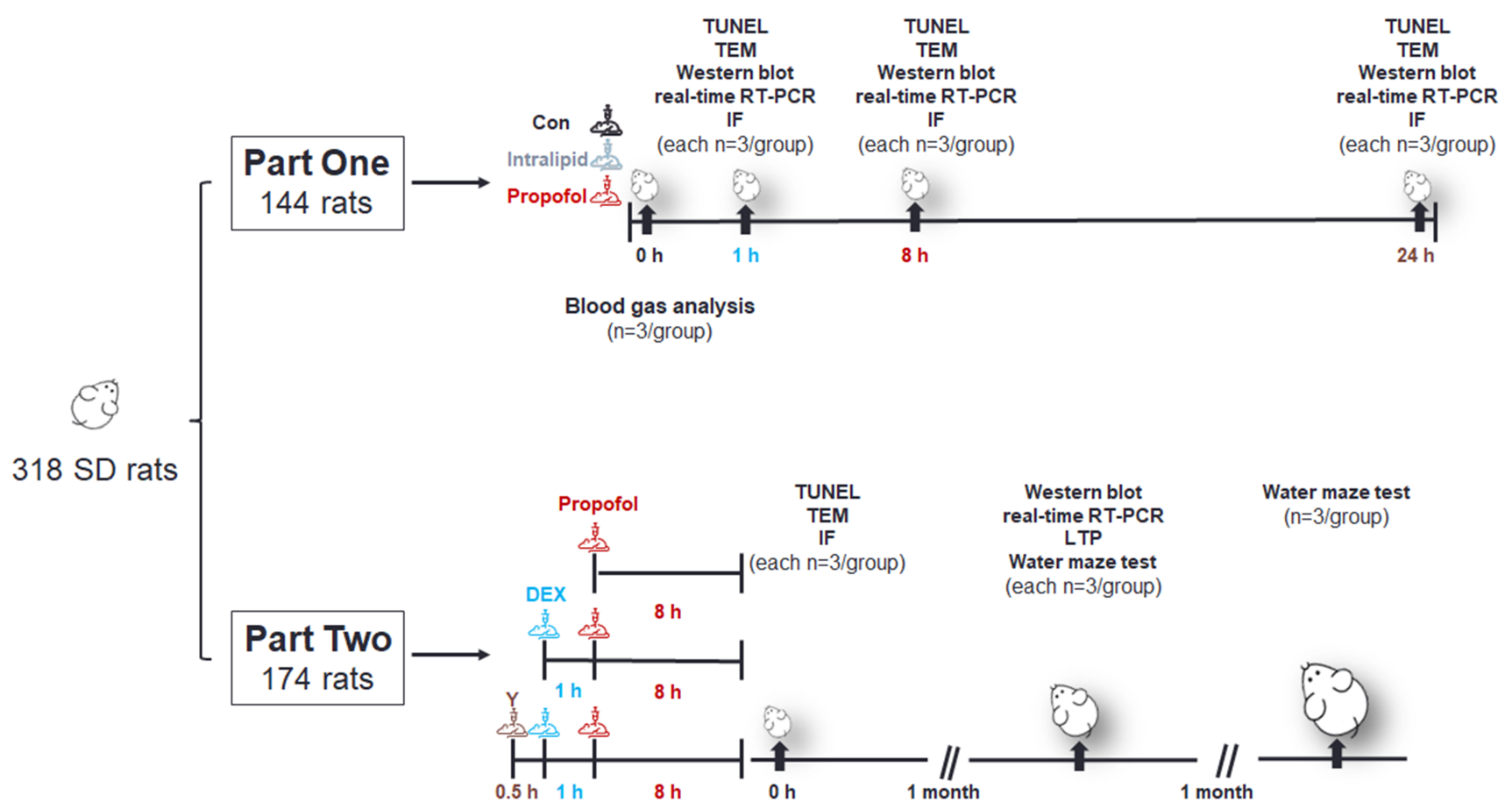

Fig. 1. Timeline of animal experimental procedures. Part 1 (144 rats): Immediately after treatment, blood gas analysis was used to exclude signs of hypoxia. TUNEL, TEM, Western blot, real-time RT-PCR and IF in P7 rats 1 h, $8 \mathrm{~h}$ and 24 h post-treatment. Part 2 (174 rats): TUNEL and TEM assays $8 \mathrm{~h}$ post-treatment (time point: $0 \mathrm{~h}$ ). Western blot, real-time RT-PCR and electrophysiological assays 1 month post-treatment. Water maze tests in 1- and 2 -month-old rats. Drugs are distinguished by different colors (brown; yohimibine, blue; DEX, red; propofol). $\mathrm{n}=3$ rats per group. Con, control; DEX, dexmedetomidine; IF, immunofluorescent staining; LTP, long term potentiation; TEM, transmission electronmicroscopy; TUNEL, Terminal deoxynucleotidyl transferase dUTP nick-end labeling; Y, yohimibine.

Recently, the potential neuroprotective effects of DEX have received wide attention. As reported previously, the neuroprotective efficacy of DEX was produced by its binding to the a2 receptor subtype [6]. Unfortunately, the potential use of DEX as an effective neuroprotectant for the prevention of neurological disorders seen in pediatric anesthesiology needs further validation. Therefore, the aim of this study was to give new insights into the effects of DEX on various propofol-mediated deficits and establish new strategies for the prevention of neurobehavioral outcomes caused by general anesthesia during early infancy.

\section{MATERIALS AND METHODS}

\section{Experimental animals and ethical statement}

All animal experimental protocols were approved by the Ethics Review Committee for Laboratory Animal Welfare of the China Medical University (Shenyang, China). Postnatal day (P) 7 Sprague Dawley male rats (10 15 g) were purchased from the Laboratory Animal Center of China Medical University, which were housed in temperature-controlled cages at $22^{\circ} \mathrm{C}$ under a $12 \mathrm{~h} \mathrm{light/dark}$ cycle (light from 06:00 to 18:00) with free access to food and water.
All protocols were performed from 8:30 10:00 and the cages were cleaned once a week. No randomization was performed. The animals were repeatedly treated according to coded numbers on their tails in sequence. All investigators who executed the analysis were blind to the groups and treatment the animals received.

\section{Study design}

Equal numbers of P7 pups (318 rats in total) were randomly assigned to one of two parts receiving different procedures (flowchart depicted in Fig. 1). Part 1: rat pups were injected intraperitoneally (i.p.) with propofol (30 mg/kg; \#16LL9315, Fresenius Kabi, Austria, Germany), in addition to an equal volume of intralipid (\#17060732, Libang Medicine Co., Ltd., Xỉan, China) or physiological saline (\#1705020511, Dubang Medicine Co., Ltd., Zhejiang, China) ( $\mathrm{n}=15$ per group). Each injection was performed with a $26-\mathrm{G}$ needle (Kangjia Medical Equipment Co., Ltd., Zhengzhou, China) to reduce pain and stress. To exclude hypoxia, three pups were used to assess blood gas levels immediately after injections (Cobas b 123, Roche, Basel, Swissland). At 1, 8, or $24 \mathrm{~h}$ post-exposure to propofol (group P1, P2 and P3), neonatal rats were anesthetized with $10 \%$ chloral hydrate $(0.35 \mathrm{ml} / 100 \mathrm{~g}$; \#47335-U, Sigma-Aldrich, 
St. Louis, MO, USA) for further experiments, including Terminal deoxynucleotidyl transferase dUTP nick-end labeling (TUNEL) assay, transmission electronmicroscopy (TEM), western blot, realtime RT-PCR, and double immunofluorescent staining (IF) ( $\mathrm{n}=3$ per group). Part 2: 174 P7 rats were randomly assigned into 11 groups: a control group (group con, $\mathrm{n}=21$ per group); a group assessed after $8 \mathrm{~h}$ i.p. post-injection of $30 \mathrm{mg} / \mathrm{kg}$ propofol (group $\mathrm{P} 2, \mathrm{n}=21$ per group); three groups receiving DEX (\#H20090248, Hengrui Medicine Co., Ltd., Jiangsu, China) at 1, 10 and $20 \mu \mathrm{g} /$ $\mathrm{kg}$ (i.p.) (group D1, n=12; group D2, n=12; and group D3, n=18); three groups receiving 1,10 , or $20 \mu \mathrm{g} / \mathrm{kg}$ DEX $1 \mathrm{~h}$ before propofol treatment, respectively (group D1+P2, n=12; D2+P2, n=12; and $\mathrm{D} 3+\mathrm{P} 2, \mathrm{n}=21)$; and groups pretreated with yohimbine ( $\mathrm{Y}$; \#HYN0127, MCE, Monmouth Junction, NJ, USA) $1 \mu \mathrm{g} / \mathrm{kg}$ (i.p.) 0.5 $\mathrm{h}$ before the similar injection regimen with DEX and propofol (group $\mathrm{Y}+\mathrm{D} 1+\mathrm{P} 2, \mathrm{n}=12 ; \mathrm{Y}+\mathrm{D} 2+\mathrm{P} 2, \mathrm{n}=12$; and $\mathrm{Y}+\mathrm{D} 3+\mathrm{P} 2, \mathrm{n}=21$ ) Three pups per group were decapitated for use in a TUNEL assay, transmission electron microscopy (TEM), and immunofluorescence (IF). We also performed Western blot and real-time RTPCR analyses on P7 rats from five groups (con, D3, P2, D3+P2, and $\mathrm{Y}+\mathrm{D} 3+\mathrm{P} 2, \mathrm{n}=3$ per group). For our electrophysiological study, at P30, three rats per group four groups (con, P2, D3+P2, and $\mathrm{Y}+\mathrm{D} 3+\mathrm{P} 2)$ were sacrificed by decapitation. The remaining $\mathrm{P} 7$ rats were used in our neurobehavioral test (water maze test) after being reared for 1 or 2 months ( $\mathrm{n}=3$ per group). Animals received no analgesics because of its potential confounding effect on the final outcome. Based on our previous experiments, we assessed the health of the animals routinely and before the start of each experiment, and no animals died or were excluded during the current set of experiments.

\section{TUNEL assay}

TUNEL assay was performed to label DNA strand breaks and was used the TUNEL assay kit (\#C1098, Beyotime Biotechnology, Beijing, China) according to the manufacturer's instructions. Briefly, paraffin embedded hippocampal slices $(5 \mu \mathrm{m})$ were deparaffinization and permeabilization, followed by incubated with TUNEL media at $37^{\circ} \mathrm{C}$ for $1 \mathrm{~h}$. The nuclei were then stained with hematoxylin (\#8070, Solarbio, Beijing, China) and washed using an ethanol gradient (\#459844, Sigma-Aldrich, St. Louis, MO, USA), and randomly chosen grids (three per slice) were analyzed. TUNEL positive cells were counted as the average apoptotic cells per high power field in a fixed grid. Then, micrographs of TUNELpositive cells were taken at $400 \times$ magnification and each test was performed in triplicate.

\section{TEM}

Rats were anesthetized i.p. with $10 \%$ chloral hydrate $(0.35 \mathrm{ml} / 100$ g; \#47335-U, Sigma-Aldrich, St. Louis, MO, USA) and perfused transcardially with $2.5 \%$ glutaraldehyde (\#A17876, Aladdin Biotechnology Co. Ltd., Shanghai, China) for a short duration (4 5 $\mathrm{min}$ ) and then brains were dissected from the skulls. The CA1 region of the hippocampi were located under light microscopy (Olympus, Tokyo, Japan), made into paraffin blocks, following the above procedure, cut into $1 \times 1 \mathrm{~mm}$ pieces with a pre-cooled blade, and fixed with 2.5\% glutaraldehyde (\#A17876, Aladdin Biotechnology Co. Ltd., Shanghai, China) in sodium cacodylate ( $\mathrm{pH}$ 7.4; \#11654, Bio-protocol, CA, USA). The slices were rinsed with sodium cacodylate for $5 \mathrm{~min}$ three times at $4^{\circ} \mathrm{C}$, dehydrated with an ethanol gradient (30 70\%, 15 min/each time) (\#459844, SigmaAldrich, St. Louis, MO, USA), and mounted after a dehydrating acetone gradient (80 100\%, $10 \mathrm{~min} / \mathrm{each}$ ) and then dried for 3 days in an oven. This was followed by embedding in fresh Epon 812 resin (\#18012, Ted pellal Inc., CA, USA). The resin-embedded specimens were cut into ultrathin sections (about $70 \mathrm{~nm}$ ) and immersed in 2\% uranyl acetate ( $\# 615-44-0$, SPI Supplies, West Chester, PA, USA) and 3\% lead citrate (\#HD17800, Hede Biotechnology, Beijing, China), and examined for cellular ultrastructure and synaptic microstructure under a high resolution scanning TEM (Hitachi H-7650, JEOL, Japan). Ten areas were randomly chosen and magnified to 7,000 diameters, $2 \times 10^{5}$ diameters, and $4 \times 10^{5} \mathrm{di}$ ameters.

\section{Real-time RT-PCR}

Total tissue RNA was extracted from hippocampi with TRIzol reagent (\#15596026, Invitrogen, Carlsbad, CA, USA) and reverse-transcribed into CDNA. cDNA was generated using the SuperScript ${ }^{\mathrm{TM}}$ III First-Strand synthesis system (\#18080051, Invitrogen, Carlsbad, CA, USA) and PCR was performed using primers specific for BCL2L1 (forward: 5'-GGACAGCATATCAGAGCTTTGAACA-3', reverse: 5'-TTGTCTACGCTTTCCACGCA-3'), matrix metalloproteinase-9 (MMP9, forward: 5'-GAATCACGGAGGAAGCCAAT-3', reverse: 5'-TAGCCCCAACTTATCCAGACT-3'), cleaved caspase-3 (CC3, forward: 5'-GACGACAGGGTGCTACGAT-3', reverse: 5'-TTTCCTTACGCTCTGACTGA-3'), and $\beta$-actin (forward: 5'-GGAGATTACTGCCCTGGCTCCTAGC-3', reverse: 5'-GGCCGGACTCATCGTACTCCTGCTT-3') using the $2^{-\Delta \Delta C t}$ method. The PCR reaction conditions were as follows: $50^{\circ} \mathrm{C}$ for $2 \mathrm{~min}$, $95^{\circ} \mathrm{C}$ for $2 \mathrm{~min}$, followed by 42 cycles at $95^{\circ} \mathrm{C}$ for $15 \mathrm{~s}$ and $60^{\circ} \mathrm{C}$ for an additional $30 \mathrm{~s}$, and each sample was run in triplicate. 


\section{Western blot}

Each fresh hippocampal specimen was collected, and total protein was extracted using radio immunoprecipitation assay (RIPA) lysis buffer (\#P0013B, Beyotime Biotechnology, Beijing, China). An equal amount of denatured protein sample $(40 \mu \mathrm{g})$ was separated by sodium dodecyl sulfate -polyacrylamide gel electrophoresis (SDS-PAGE) and transferred to polyvinylidene fluoride (PVDF; IPVH00010, Millipore, MA, USA) membranes. The PVDF membranes were probed with primary antibodies against BCL2L1 (1:1,000; \#bs-1336R, Bioss, Woburn, MA, USA), MMP-9 (1:1,000; \#bs-4593R, Bioss, Woburn, MA, USA), CC3 (1:1,000; \#9661, CST, Danvers, MA, USA) and $\beta$-actin (1:3,000; \#A5441, Sigma-Aldrich, St. Louis, MO, USA), diluted in blocking buffer overnight at $4^{\circ} \mathrm{C}$. The next day following three washes with Tris-buffered saline containing 0.1\% Tween-20 (\#93773, Sigma-Aldrich, St. Louis, MO, USA), the blots were probed with secondary antibody labeled with horseradish peroxidase-conjugated goat anti-rabbit $\operatorname{IgG}(1: 4,000$; \#7074, CST, Danvers, MA, USA) for $2 \mathrm{~h}$ at room temperature. Protein bands were visualized using an enhanced chemiluminescent kit (\#32106, Thermo Fisher Scientific, Waltham, MA, USA). The intensity of each band was quantified with a computer-assisted image analysis system (NIH Image Analysis, Bethesda, MD, USA) and normalized to the intensity of $\beta$-actin. All experiments were repeated three times.

\section{In-vivo double immunofluorescent staining of P7 hippo- campal astrocytes}

Hippocampi were removed and prepared for dehydration using graded ethanol (70 90\%), and immersion fixation in the mixture of dimethylbenzene and wax. Paraffin blocks were then cut into 5 $\mu \mathrm{m}$ sections and all slides were dried in an incubator at $60^{\circ} \mathrm{C}$ for 2 $\mathrm{h}$, then dewaxed for $30 \mathrm{~min}$ and incubated in blocking buffer for $30 \mathrm{~min}$ at room temperature $\left(20 \sim 24^{\circ} \mathrm{C}\right)$. Next primary antibodies against glial fibrillary acidic protein (GFAP, 1:50; Santa Cruz Biotechnology, Santa Cruz, CA, USA) and BCL2L1, MMP-9 and CC3 (1:100; Bioss Technology, Beijing, China) were added and sections were incubated at $4^{\circ} \mathrm{C}$ overnight. After multiple rinses in phosphate-buffered saline, the sections were blocked with goat antimouse IgG and goat anti-rabbit IgG (1:200; Beyotime Institute of Biotechnology, Shanghai, China) and all the sections were stained with 4,6-diamidino-2-phenylindole (DAPI, Biosharp, Carlsbad, CA, USA) at room temperature $\left(20 \sim 24^{\circ} \mathrm{C}\right)$. For double immunofluorescent analysis, the sections were imaged and photographed using a laser scanning confocal microscopy (Olympus, Tokyo, Japan).

\section{LTP recording}

After each injection of $10 \%$ chloral hydrate $(0.35 \mathrm{ml} / 100 \mathrm{~g}$; \#47335-U, Sigma-Aldrich, St. Louis, MO, USA), the anesthetized $\mathrm{SD}$ rats (P30 of age) were decapitated immediately. The brains were removed from the skull and quickly placed in the icecold oxygenated artificial cerebrospinal fluid (aCSF) containing (in mM, pH 7.4) $124 \mathrm{NaCl}$ (\#106406, Sigma-Aldrich, St. Louis, MO, USA), 3KCl (\#P3911, Sigma-Aldrich, St. Louis, MO, USA), $26 \mathrm{NaHCO}_{3}$ (\#S6014, Sigma-Aldrich, St. Louis, MO, USA), 2 $\mathrm{CaCl}_{2}$ (\#499609, Sigma-Aldrich, St. Louis, MO, USA), $1 \mathrm{MgSO}_{4}$ (\#746452, Sigma-Aldrich, St. Louis, MO, USA), $1.25 \mathrm{KH}_{2} \mathrm{PO}_{4}$ (\#795488, Sigma-Aldrich, St. Louis, MO, USA) and 30Glucose (\#G8270, SigmaAldrich, St. Louis, MO, USA). Coronal $400 \mu \mathrm{m}$-thick hippocampal slices were cut in ice-cold aCSF using an automatic vibrate microtome (VT 1200 S, Leica, Nussloch, Germany) and transferred into a chamber containing aCSF pregassed with $95 \% \mathrm{O}_{2} / 5 \% \mathrm{CO}_{2}$ at room temperature for $1 \mathrm{~h}$. The electrophysiological recording in acute hippocampal slices, which continuously superfused with well oxygenated saturated aCSF (at a rate of $1.0 \mathrm{ml} \cdot \mathrm{min}^{-1}$ at room temperature) throughout the electrophysiological period, was laid down on a 64-channel multi-microelectrode array (MED-P515A, Alpha MED Sciences, Panasonic, Japan). The placement of each slice was quickly adjusted in the probe under an inverted microscopy (Leica, Germany, $\times 40$ times).

During stimulation, all signals were acquired by the MED64 Mobius system (Alpha MED Sciences, Panasonic, Japan). The appropriate stimulation site and amplitude were identified in response to increasing intensities from $8 \mu \mathrm{A}$ to $30 \mu \mathrm{A}$ (in $2 \mu \mathrm{A} /$ step) to abtain input-output (I/O) curves. After the basic field excitatory postsynaptic potentials (fEPSPs) was stably recorded for $30 \mathrm{~min}$, LTP was evoked by applying theta burst stimulus (TBS), ten trains repeated, each train consisted of five bursts separated by $200 \mathrm{~ms}$, each burst consisted of four biphasic pulses at $100 \mathrm{~Hz}$, with the stimulation intensity which could elicit 30 50\% of the maximal current amplitude in the electrode. Only slices showing stable baseline were recorded after TBS application for $1 \mathrm{~h}$. For each slice, if the slope was increased by $30 \%$ (or more) of the maximum fEPSP amplitude and sustained for $20 \mathrm{~min}$, the response was taken as LTP. MED64 Mobius 0.5.0 software (Alpha MED Sciences, Panasonic, Japan) collected data and measured the population spike (PS) amplitude and fEPSP slope. The size of PS was measured between the peak and the trough of the negative potential. The slope of fEPSP was recorded and calculated in the initial linear part of each potential. The results were normalized to the percentage change from the baseline, which was the mean value of corresponding amplitude (or slope) $30 \mathrm{~min}$ before TBS stimulation. 
Table 1. Blood gas analysis of P7 rats tested immediately after saline, intralipid or propofol $30 \mathrm{mg} / \mathrm{kg}$ i.p injection

\begin{tabular}{lccccc}
\hline Group & $\mathbf{p H}$ & $\mathbf{P a O}_{\mathbf{2}} \mathbf{( \mathbf { m m H g } )}$ & $\mathbf{P a C O}_{\mathbf{2}} \mathbf{( \mathbf { m m H g } )}$ & $\mathbf{H C O}_{\mathbf{3}}{ }^{-} / \mathbf{m m o l}^{-\mathbf{L}^{-1}}$ & $\mathbf{B E}$ \\
\hline Control & $7.30 \pm 0.07$ & $93.7 \pm 9.1$ & $42.1 \pm 5.3$ & $27.7 \pm 5.1$ & $-2 \pm 1$ \\
Intralipid & $7.30 \pm 0.01$ & $92.8 \pm 10.1$ & $43.5 \pm 5.2$ & $28.8 \pm 4.1$ & $-2 \pm 1$ \\
Propofol & $7.31 \pm 0.02$ & $94.3 \pm 6.2$ & $40.6 \pm 5.1$ & $27.8 \pm 4.6$ & $-2 \pm 1$ \\
\hline
\end{tabular}

Data are shown as mean $\pm \mathrm{SD}$ ( $\mathrm{n}=3$ each group).

$\mathrm{PaO}_{2}$, arterial oxygen tension; $\mathrm{PaCO}_{2}$, arterial carbon dioxide tension. $\mathrm{p}>0.05$.

\section{Water maze test}

The water maze test is an important tool used to measure the spatial learning and hippocampus-dependent memory of laboratory animals [7]. The water maze test was performed at P30 and P60, according to standard procedures as previously described [8]. The experiment was performed on six consecutive days, including one day of visible platform trial, four days of hidden platform trial, and one day of probe trial. The water maze consisted of a round tank with a diameter of $160 \mathrm{~cm}$, filled with water at $25 \pm 1^{\circ} \mathrm{C}$. The performance of the rats in the water maze was tracked and recorded automatically using EthoVision XT software (Noldus, Leesburg, VA, USA).

\section{Statistical analysis}

All statistical analysis was performed by GraphPad Prism 7.0 (GraphPad Software Inc., La Jolla, USA). Data are presented as mean \pm standard deviation (SD) of three independent experiments. Differences among multiple groups were compared by one-way analysis of variance (ANOVA), followed by Tukey's test. In the LTP test, data of pre-TBS and post-TBS was compared by unpaired Student's $t$-test in same group. In water maze trial, repeatedmeasures ANOVA was used to analyze data of hidden platform trial. Meanwhile, data obtained from probe trial was analyzed by one-way ANOVA. $\mathrm{p}<0.05$ was considered statistically significant difference.

\section{RESULTS}

\section{Blood gas analysis showed no hypoxia}

No changes in $\mathrm{pH}, \mathrm{PaO}_{2}, \mathrm{PaCO}_{2}$, and $\mathrm{HCO}_{3}{ }^{-}$were seen in the propofol-exposed group when compared to the control, or intralipid groups $(\mathrm{p}>0.05)$ (Table 1). This finding indicated that there was no significant hypoxia or hypercapnia in the P7 rats after a single propofol $(30 \mathrm{mg} / \mathrm{kg})$ treatment.

\section{Propofol promoted apoptosis in hippocampal neurocytes and synaptic structural modification in $P 7$ rats}

When compared to control or intralipid group, propofol significantly increased the number of TUNEL positive cells in the
CA1 region at 1,8 , or $24 \mathrm{~h}$ following a single propofol treatment in vivo $\left({ }^{* * *} \mathrm{p}<0.0001\right)$ (Fig. $\left.2 \mathrm{~A}\right)$. These data suggested that neonatal propofol exposure exerted a toxic effect on the developing neurocytes. Furthermore, to interpret the apoptotic ultrastructural morphological changes, representative images of neurocytes and synapses from P7 rodent hippocampal CA1 regions were examined under TEM (Fig. 2B). In the control group, both in neurons and astrocytes, chromatin was evenly distributed in regular nuclear shapes and the cells had intact nuclear membranes (Fig. $2 \mathrm{Ba}$ ). In the intralipid group, the astrocytes, contained filaments and a clear boundary around the organelles in the cytoplasm and surrounding the synapses (Fig. 2Bb); this plays a vital role not only in supplying nutrients to neurons, but also facilitating synaptic plasticity [9]. Both phenomena are related to the process of reshaping the developing mammalian brain. In the P1 group, the nuclear chromatin migrated to the nuclear envelope, which had the appearance of a moon or clock like shape. The nuclear envelope was not smooth but folded, but nevertheless remained intact, with condensed organelles and a deeply stained cytoplasm (Fig. 2Bc). At 8 h post- injection of propofol (30 mg/kg, group P2), astrocyte nuclear membranes fragmented with obvious chromatin condensation, forming a ring-like, electron-dense pyknotic mass (Fig. 2Bd). In the P3 group, a more advanced stage of apoptosis was seen, characterized by the destruction of the nuclear membrane and clumping nuclear chromatin. The mitochondria were ill defined with a homogeneous density and total absence of cristae (Fig. $2 \mathrm{Be}$ ). Furthermore, the synaptic ultrastructure in P7 rodent CA1 regions is shown in Fig. $2 \mathrm{Bf} \sim \mathrm{j}$. In both the control and intralipid groups, the synaptic clefts were clear with intact morphology (Fig. $2 \mathrm{Bf}$ and $2 \mathrm{Bg}$ ), and multiple synaptic vesicles (SVs) could be seen, which released into the synaptic cleft. In addition, the postsynaptic density (PSD) was abundant and had a homogenous distribution. In the P1 group, the number of presynaptic vesicles decreased (Fig. 2Bh). In the P2 group, there was a decrease in vesicles and the synaptic cleft became undefined (Fig. 2Bi). After a 24 h exposure to propofol in group P3, the synaptic morphology was destroyed with a wider cleft, blurred boundaries, and fewer vesicles (Fig. 2Bj). 


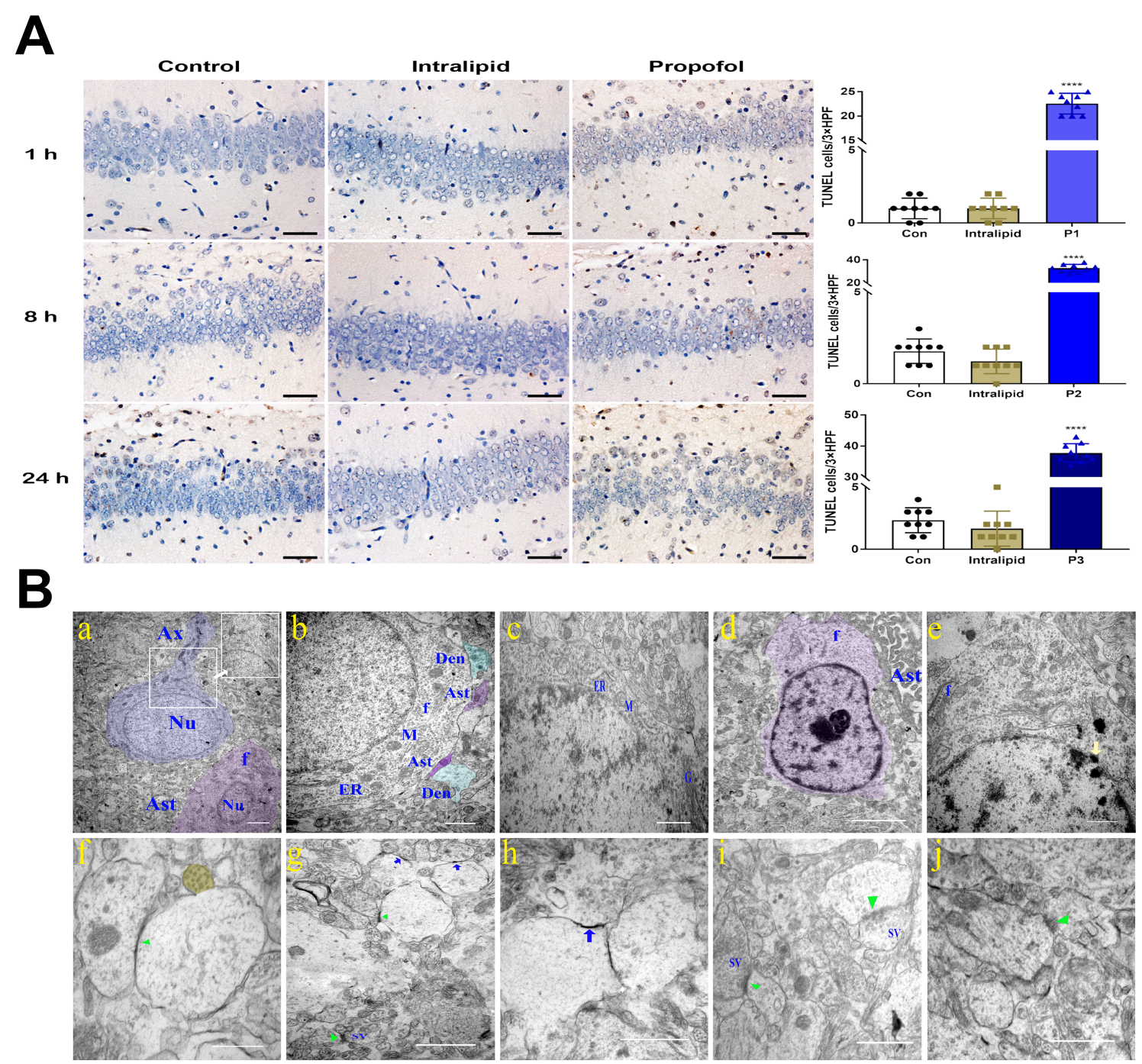

Fig. 2. Effect of neonatal propofol exposure on immature hippocampal neurocytes and synapse. (A) TUNEL staining showing the number of apoptotic cells, $n=9$ slices per group, from three rats. Scale bar=50 $\mu \mathrm{m}$. (B) TEM images of the rodent hippocampi. After termination of the 1-, 8- and 24hour exposure to $30 \mathrm{mg} / \mathrm{kg}$ propofol, which was designed as P1, P2 and P3 group, hippocampal ultrastructure was evaluated under a scanning TEM. (a) Neurons (blue) and astrocytes (Ast, purple) in the control group. Axon, Ax; filament, f; nucleus, Nu. Scale bar=2 $\mu \mathrm{m}, 1 \mu \mathrm{m}$ in the right upper rectangle (magnified picture). (b) In the intralipid group, dendrite (Den, blue), endoplasmic reticulum (ER), and mitochondria (M) are visible. Scale bar=1 $\mu$ m. (c) In the P1 group, M shows homogeneous densities with relatively well-shaped cristae, occasionally with vacuoles inside. Some rough ER is swollen. Golgi apparatus, G. Scale bar=1 $\mu \mathrm{m}$. (d) In the P2 group, Ast (purple) containing multiple f and an obvious condensed nuclear chromatin with intact nuclear envelop is highlighted. Scale bar $=2 \mu \mathrm{m}$. (e) Destruction of nuclear membrane in the P3 group (yellow arrow). Scale bar=1 $\mu \mathrm{m}$. (f) In the control group, the synaptic ultra-structural morphology of rodent hippocampal CA1 regions was intact with clear synaptic vesicles (SVs, yellow), green arrow heads: asymmetric synapse. Scale bar=500 nm. (g) In the intralipid group, both asymmetric (green arrowheads) and symmetric synapses (blue arrow) with dense postsynaptic density (PSD) are shown. Scale bar=1 $\mu \mathrm{m}$. (h) In the P1 group, the synaptic ultrastructure is still complete with fewer SVs. Scale bar=1 $\mu \mathrm{m}$. (i) In the P2 group, the synaptic cleft is obsured but still visible PSD. Scale bar=1 $\mu \mathrm{m}$. (j) In the P3 group, the cleft of the asymmetric synapse (green arrowheads) grew wider with blurred boundaries and fewer SVs. Scale bar $=1 \mu \mathrm{m} . \mathrm{n}=3$ animals per group. All data are presented as mean \pm SD from three independent experiments. ${ }^{* * *} \mathrm{p}<0.0001$ vs. the control group.

\section{The effect of propofol on MMP-9/BCL2L1/CC3 signaling in P7 rats}

To quantify changes in MMP-9, BCL2L1, and CC3 expression levels, both mRNA and proteins from P7 rodent hippocampi were tested. The level of MMP-9 was significantly downregulated after propofol treatment when compared to controls (Fig. 3A). Moreover, propofol also reduced the expression level of BCL2L1 (Fig. 3B). However, the levels of the apoptotic terminal executioner CC3, at both the mRNA and protein levels in hippocampi from P7 rodents were significantly increased after a single propofol injec- 
A

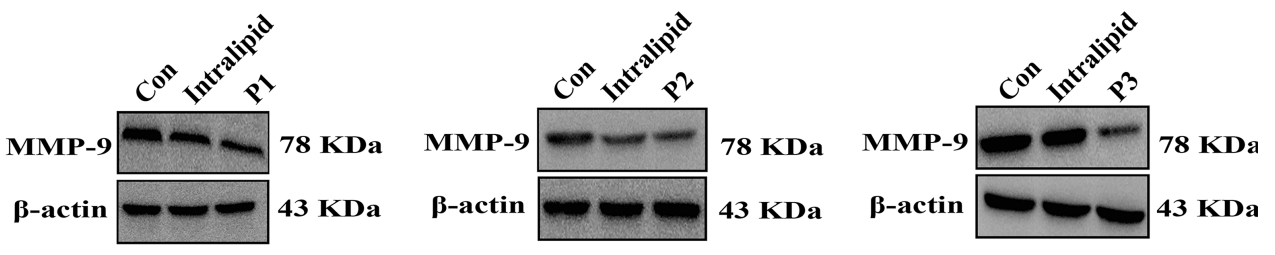

- Con a Intralipid $\mathbf{v}$ Propofol
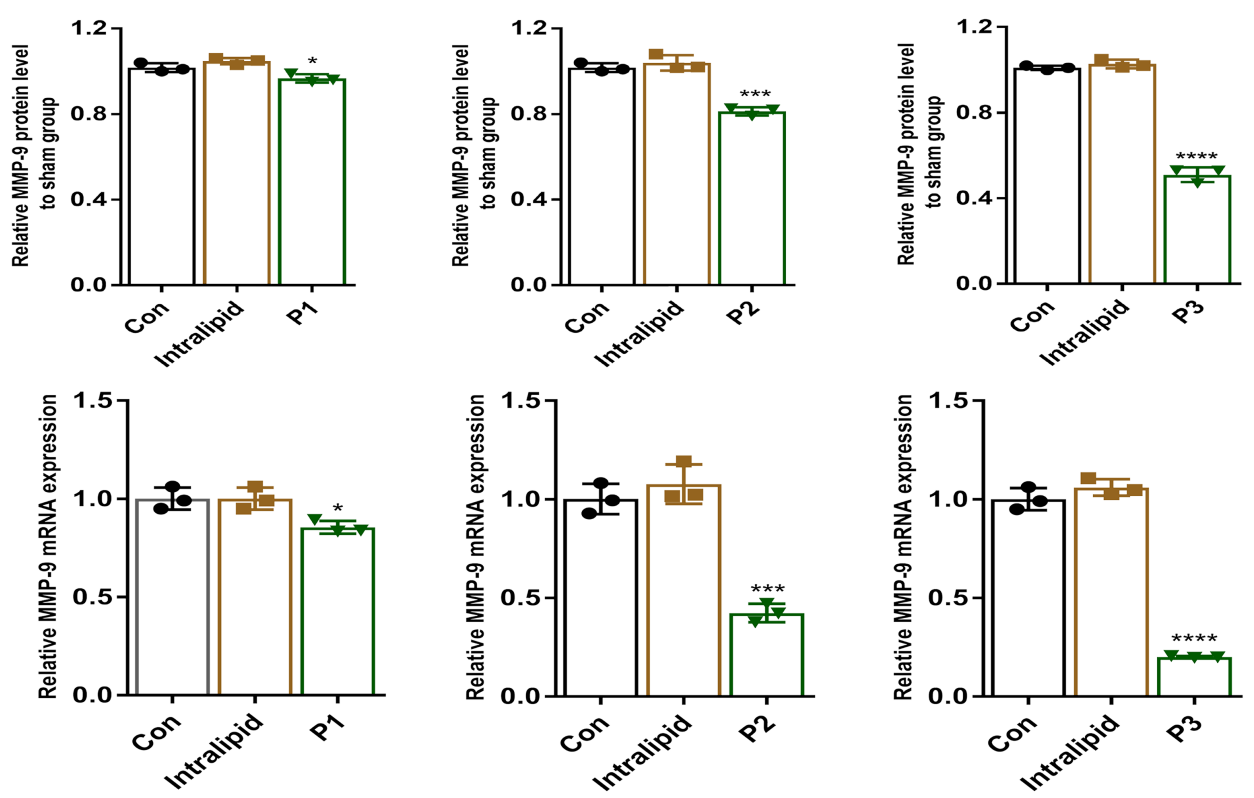

B

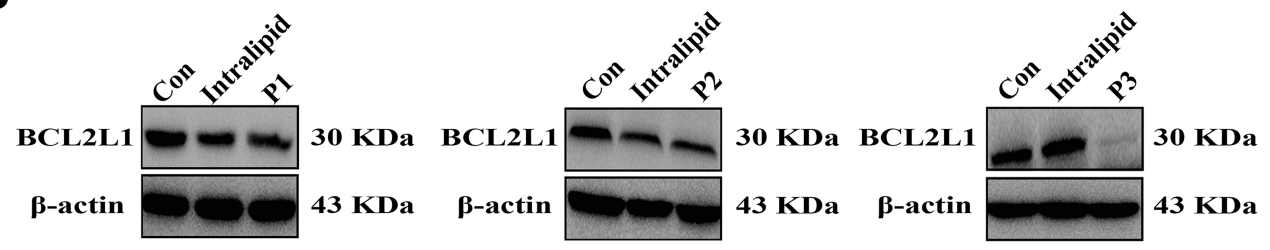

- Con = Intralipid $\Delta$ Propofol
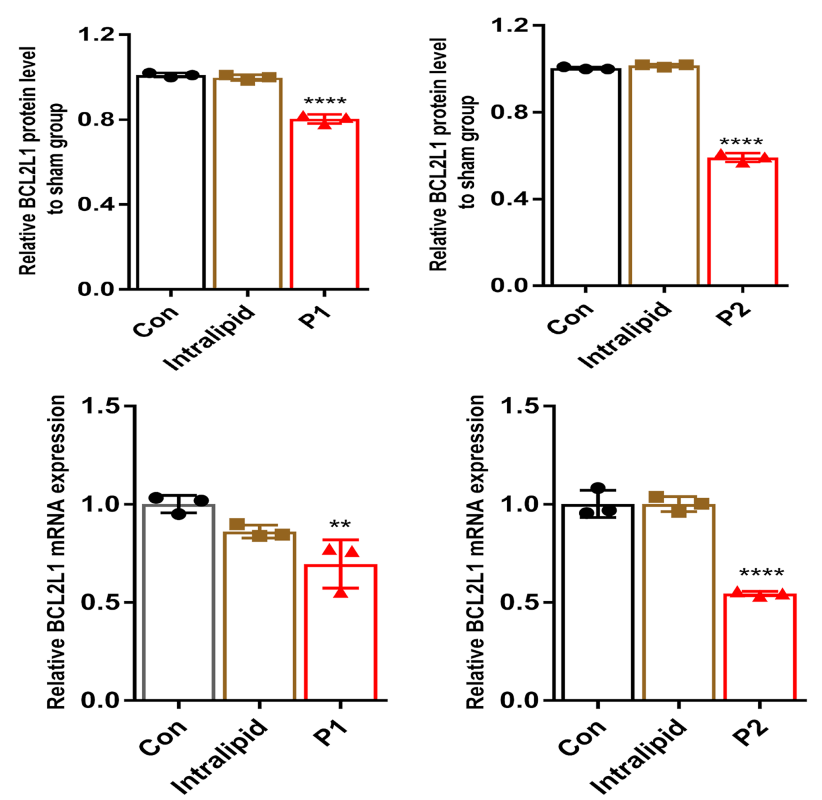
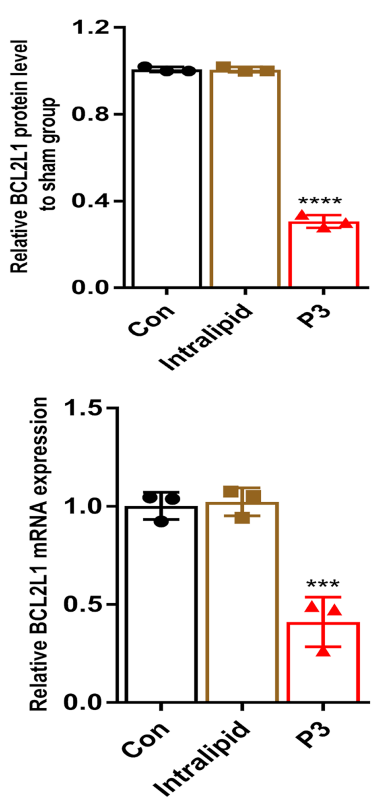

Fig. 3. Expression levels of MMP-9, BCL2L1 and CC3. Western blot and quantitative RT-PCR for A, MMP-9, B, BCL2L1, and C, CC3. $\beta$-actin was used as internal control. Groups which were exposed to $30 \mathrm{mg} / \mathrm{kg}$ propofol after termination of 1,8 and $24 \mathrm{~h}$ were designated as P1, P2 and P3 group, seperately. Results are expressed as multiples relative to the controls (mean $\pm \mathrm{SD}$ ). $\mathrm{n}=3$ animals per group. Each test was repeated three times. ${ }^{*} \mathrm{p}<0.05,{ }^{* *} \mathrm{p}<0.01$, ${ }^{* * *} \mathrm{p}<0.001,{ }^{* * * *} \mathrm{p}<0.0001$ vs. the control group. 
C

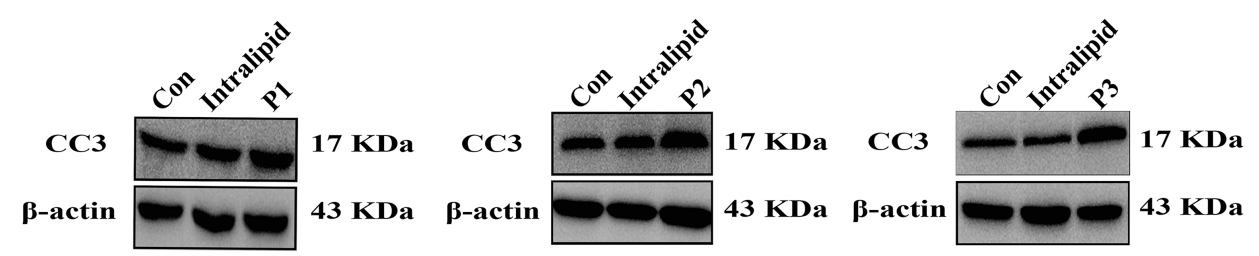

- Con - Intralipid - Propofol
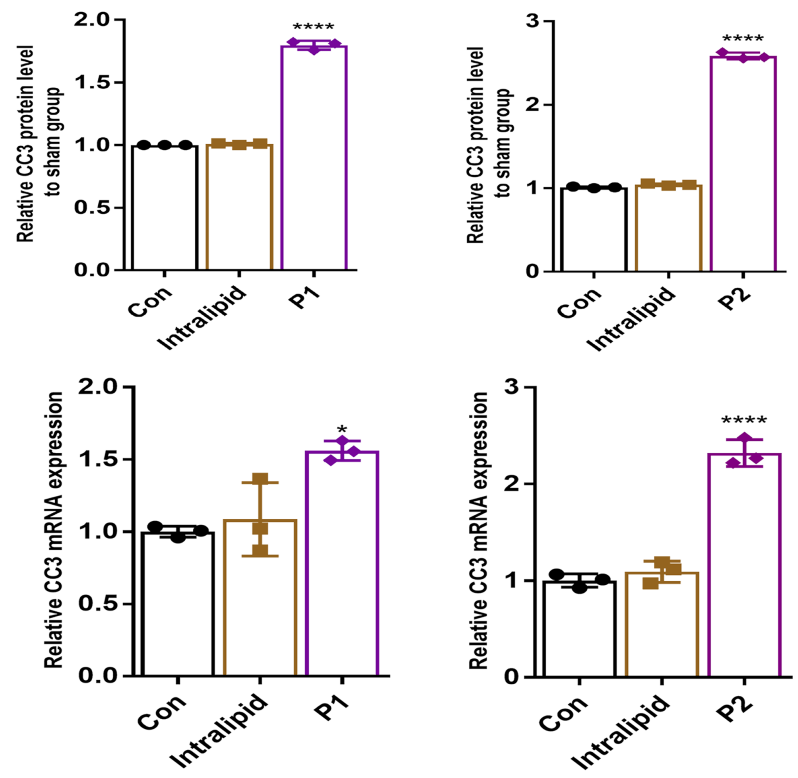
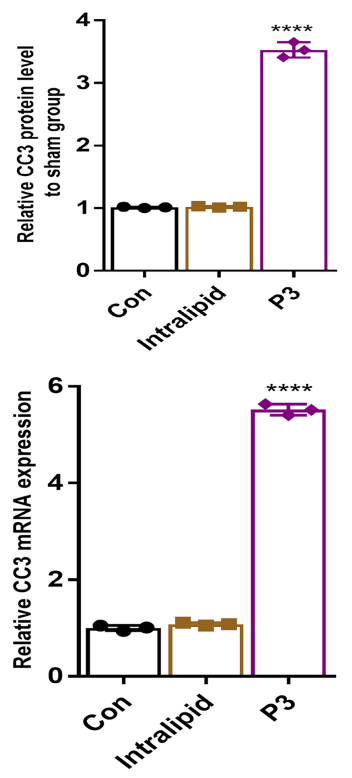

Fig. 3. Continued.

tion (Fig. 3C).

IF assays revealed that propofol time-dependently suppressed the protein expression of BCL2L1, with a $29.15 \%$ decrease at $1 \mathrm{~h}$, a $66.60 \%$ decrease at $8 \mathrm{~h}$ and a $96.61 \%$ decrease at $24 \mathrm{~h}$. Whereas, the percentage levels for MMP-9 were $14.84 \%, 42.31 \%$ and $73.53 \%$, for the same time points. However, the immunostaining intensity for CC3 in P7 rodent hippocampal slices were significantly exacerbated after 1 h, 8 h or 24 h by i.p. injection of propofol, accounting for $10.43 \%, 54.87 \%$ and $160.97 \%$ reductions (Fig. 4A C). Taken together, these data suggest that apoptosis was exacerbated by propofol in newborn rat hippocampi.

\section{DEX rescued propofol-induced apoptosis}

Pretreatment with DEX 10 and $20 \mu \mathrm{g} / \mathrm{kg}$ prior to the addition of propofol attenuated the number of TUNEL positive cells. However, in the $\mathrm{Y}+\mathrm{D} 3+\mathrm{P} 2$ group, DNA strand breaks were increased, demonstrating that the anti-apoptotic effect of DEX was inhibited by use of the $a 2$-adrenoceptor antagonist yohimbine $(p=0.0007)$ (Fig. 5).

Representative micrographs depicting cellular and synaptic ultrastructure of the CA1 region of the newborn rats are shown in
Fig. 6. The cellular apoptosis-like features were revealed in the nucleus after propofol exposure, including abnormal nuclear shape, accompanied by increased heterochromatin agglutination (Fig. $6 \mathrm{~B}$ and $6 \mathrm{C}$ ). Fig. $6 \mathrm{D} \sim \mathrm{F}$ shows the normal structure of synapses and astrocytes in the D1, D2, and D3 groups, respectively. As the dose of DEX increased, the structural destruction of the synapse recovered, as revealed by an increase in SVs and a narrowing of the synaptic cleft, with dense PSD (Fig. 6G I). However, in the $\mathrm{Y}+\mathrm{D} 1 /$ $\mathrm{D} 2 / \mathrm{D} 3+\mathrm{P} 2$ groups, the situation once again deteriorated, as fewer SVs and an obscure boundary of the synaptic membranes were seen (Fig. 6J L).

To identify the influence of DEX on propofol induced apoptosis, the distribution and intensity of IF for the apoptosis related genes was assessed. The fold changes in intensity of BCL2L1 and MMP-9 in the single propofol group were remarkably reduced in GFAP-positive astrocytes when compared to the control group, along with the distinctly exaggerated immunoreactive CC3 coexpression $(\mathrm{p}<0.0001)$. DEX at different concentrations had no apparent effect on the intensity ( $p>0.05)$, but when propofol was incorporated with DEX, DEX inhibited the effect on propofol toxicity, which enhanced the intensity of BCL2L1 and MMP-9 stain- 


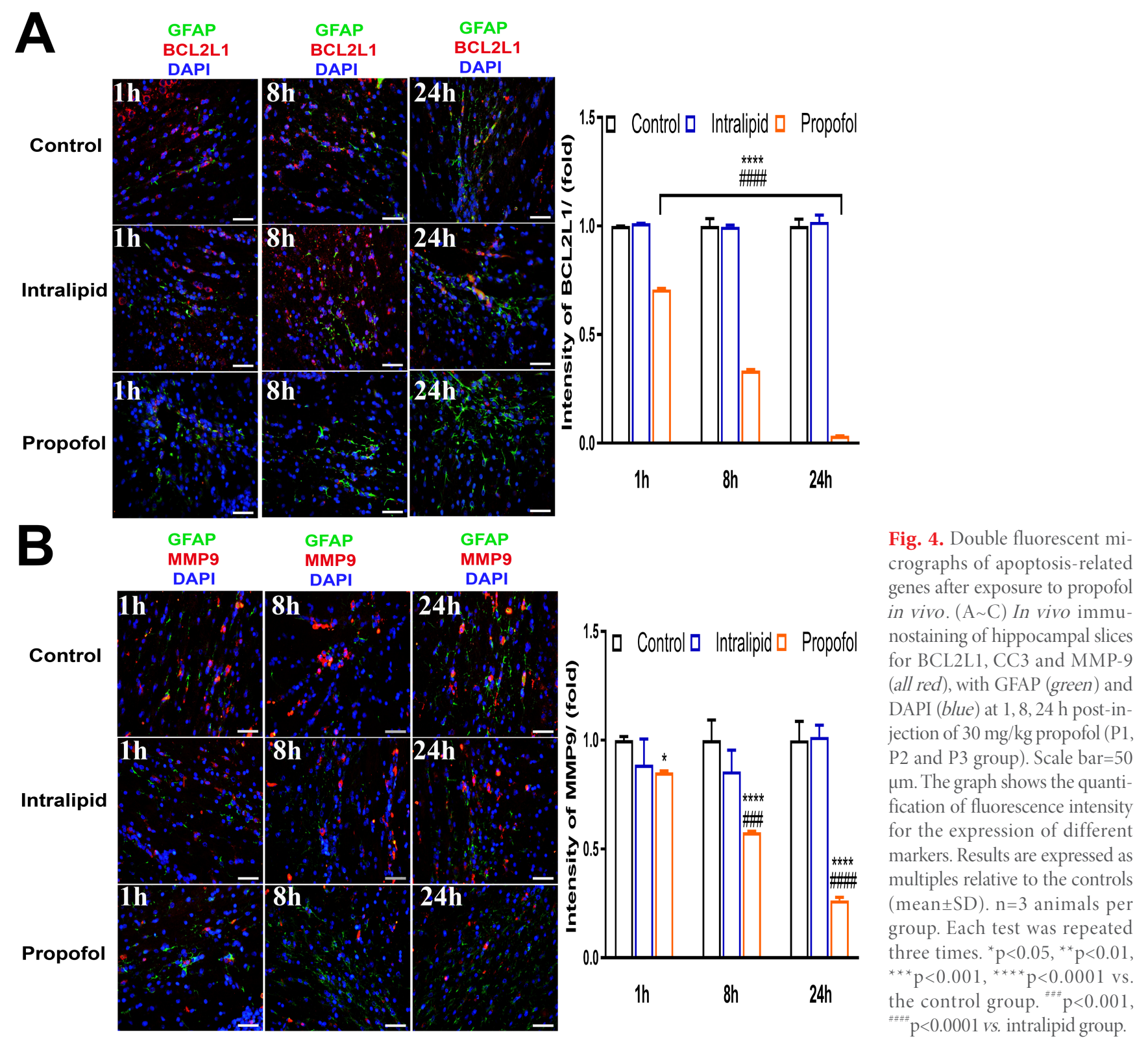

ing. In comparison, after yohimbine treatment, the mitigating effect of DEX was reversed ( $p<0.001$, Fig. 7). Therefore, DEX appears to play a protective role in the apoptosis-related signaling initiated by propofol. Furthermore, yohimbine was sufficient to counter the neuroprotection afforded by DEX in the developing brain in vivo, after co-treatment with propofol.

\section{DEX inhibited propofol-induced synaptic plasticity deficits and apoptotic signaling in $P 30$ rats}

To determine the therapeutic effects of DEX, we used a dose of $20 \mu \mathrm{g} / \mathrm{kg}$ and evaluated its influence on long-term synaptic plasticity following single propofol injection. We recorded the evoked potentials coronal hippocampal slices (Fig. 8A). The theta burst stimulation (TBS)-induced LTP was maintained over $1 \mathrm{~h}$ and there was no significant difference in the stimuli intensity. Intralipid application showed no effect on the baseline field excitatory post-synaptic potentials (fEPSPs) and TBS-induced LTP prior to experimentation ( $\mathrm{p}>0.05)$.

TBS induced a typical LTP in hippocampal CA1 of P30 rats. TBS elicited a robust LTP in the control group (pre-TBS 100.0 \pm 4.7 normalized amplitude vs. post-TBS $239.0 \pm 4.8 \%, \mathrm{t}=226.9, \mathrm{p}<0.0001$; pre-TBS $100.0 \pm 4.4$ normalized slope vs. post-TBS $526.7 \pm 13.0 \%$, $\mathrm{t}=309.9, \mathrm{p}<0.0001$ ). Whereas, propofol significantly abrogated this hippocampal synaptic plasticity (pre-TBS 100.0 \pm 5.5 normal- 


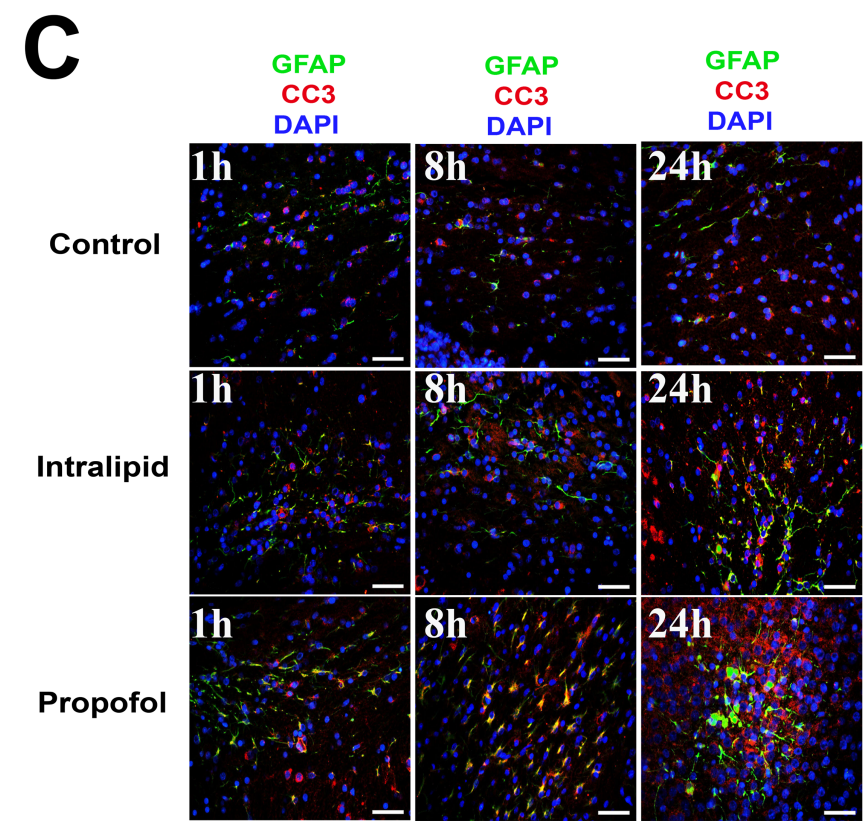

ized amplitude vs. $185.2 \pm 8.5 \% ; \mathrm{t}=86.3, \mathrm{p}<0.0001)$. By not affecting the basic synaptic transmission or LTP formation, the PS slope of fEPSP was increased up to $346.3 \pm 9.0 \%$ in the P group (postTBS $102.9 \pm 25.2 \%, \mathrm{t}=115.6, \mathrm{p}<0.0001)$. The propofol-impaired synaptic plasticity was recovered considerably by pre-treatment with DEX (pre-TBS 99.8 \pm 5.2 normalized amplitude vs. post-TBS $800.2 \pm 10.6 \%$; $\mathrm{t}=606.7, \mathrm{p}<0.0001$; pre-TBS $99.8 \pm 5.3$ normalized slope vs. post-TBS $1319.0 \pm 95.6 \%, \mathrm{t}=124.2, \mathrm{p}<0.0001)$. Yohimbine, the specific inhibitor of DEX, conduced to a reduced LTP response (pre-TBS 100.0 \pm 4.5 normalized amplitude vs. 486.6 $\pm 5.0 \%$; $\mathrm{t}=624.1, \mathrm{p}<0.0001)$ and increased LTP slope in the $\mathrm{Y}+\mathrm{D} 3+\mathrm{P}$ group (pre-TBS $100.1 \pm 4.8$ normalized slope vs. post-TBS $705.6 \pm 7.7 \%$, $\mathrm{t}=697.9, \mathrm{p}<0.0001)$

Injection of $30 \mathrm{mg} / \mathrm{kg}$ propofol markedly suppressed the increase in LTP after TBS. In the P group, the overall mean fEPSP amplitude and slope were $136.8 \pm 3.7 \%$ and $259.5 \pm 7.2 \%$, seperately $\left({ }^{* * *} \mathrm{p}<0.0001\right.$ vs. the control group). In the $\mathrm{D} 3+\mathrm{P}$ group, pre-treatment with DEX exhibited a significant enhancement in averaged amplitude (800.2 $\pm 10.6 \%)$ and slope (893.5 $\pm 35.6 \%)(\# \# \# \mathrm{p}<0.0001$ vs. P group), which implied the reversal of the propofol-induced depression of LTP was owing to the DEX pre-treatment. In $\mathrm{Y}+\mathrm{D} 3+\mathrm{P}$ group, the mean amplitude of fEPSPs and slope were significantly depressed to $486.6 \pm 5.0 \%$ and $491.9 \pm 17.6 \%$ respectively ( ${ }^{\& \& \& 8} \mathrm{p}<0.0001$ vs. D3+P group) (Fig. 8B and 8C).

After neonatal exposure to general anesthetics, the expression of BCL2L1 in the hippocampi of P30 rodents was inhibited by neonatal propofol exposure. When compared to the $\mathrm{P}$ group, data revealed a significant increase in BCL2L1 mRNA and protein in

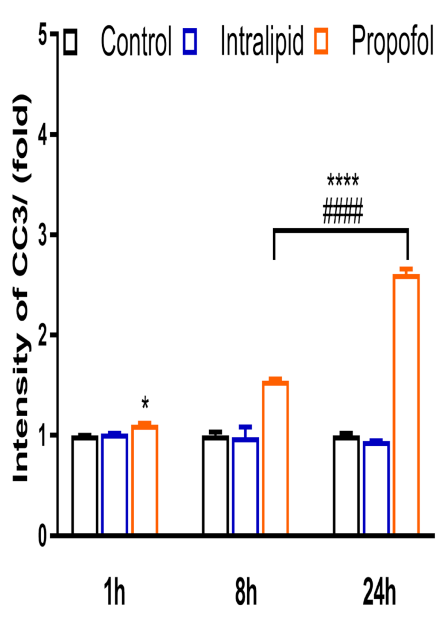

Fig. 4. Continued.

the $\mathrm{D} 3+\mathrm{P}$ group. However, in the $\mathrm{Y}+\mathrm{D} 3+\mathrm{P}$ group, BCL2L1 expression was suppressed. This was similar to the MMP-9 mRNA and protein changes seen in the $\mathrm{D} 3+\mathrm{P}$ group and the $\mathrm{Y}+\mathrm{D} 3+\mathrm{P}$ group. However, the expression of CC3 mRNA and protein was dramatically increased in $\mathrm{P} 30$ rodent hippocampal samples. The protein expression of CC3 was downregulated in the $\mathrm{D} 3+\mathrm{P}$ group but was enhanced after yohimbine treatment (Fig. 8D and 8E).

\section{A single exposure to propofol in neonates was insufficient to impair juvenile spatial learning and memory}

Different groups of anesthetic treated and control rats were subjected to the water maze, one or two months after drug injection (i.p.) to $\mathrm{P} 7$ rats ( $\mathrm{n}=3$ per group). In the acquisition phase, the anesthetic treated groups showed no significant decline in escape latency in reaching the hidden platform (quadrant III). The first color image shows the escape latency of 1-month-old rats $\left(\mathrm{F}_{(30,60)}=1.71\right.$, $\mathrm{p}=0.0385)$ (Fig. 9B) and 2-month-old rats $\left(\mathrm{F}_{(30,60)}=0.931, \mathrm{p}=0.5752\right)$ (Fig. 9E). The probe trial confirmed our previous findings that the anesthesia-exposed groups of juvenile rats exhibited control-like performance in locating the escape platform, which was removed from the exact location in the target quadrant $(\mathrm{p}>0.05)$ (Fig. 9B and 9E). No statistical differences in crossing times in the target quadrant were seen in the 1 - and 2-month-old rats $\left(\mathrm{F}_{(10,22)}=0.95\right.$ and 1.681, $\mathrm{p}=0.51$ and 0.15 ). In addition, no statistical differences in platform crossing times were seen in the 1- and 2-month-old rats $\left(\mathrm{F}_{(10,22)}=1.78\right.$ and $1.61, \mathrm{p}=0.13$ and 0.17$)$. The latency to first crossing plateau of 1- and 2-month-old rats were not obvious increased $\left(\mathrm{F}_{(10}\right.$, ${ }_{22}=3.85$ and $0.87, \mathrm{p}=0.06$ and 0.57 , respectively). Similar levels in the 


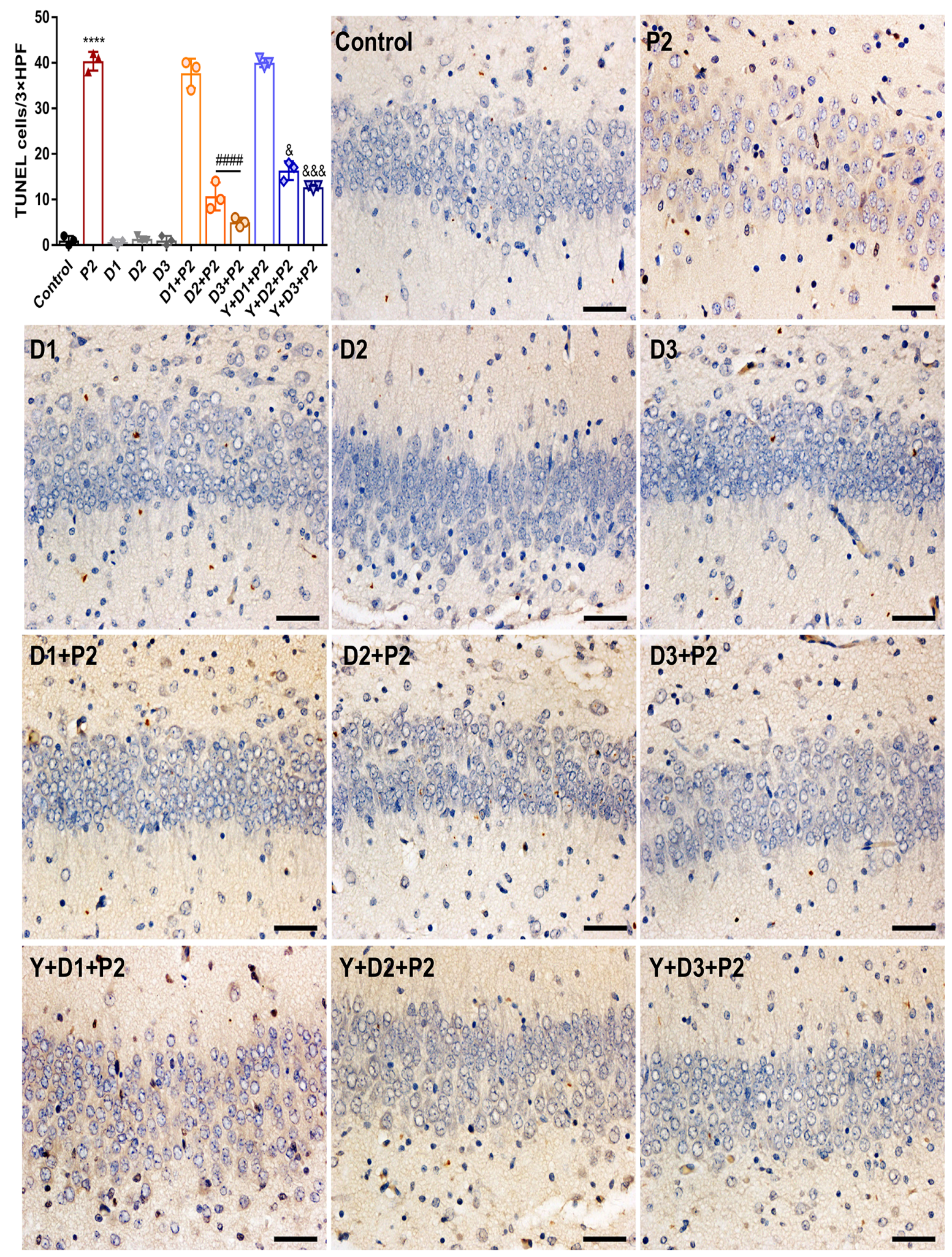

Fig. 5. Protective effect of DEX on P7 hippocampal injury induced by a single dose of propofol. TUNEL staining in the hippocampal CA1 area of rodent pups. The protective effects of dexmedetomidine (DEX) were inhibited by yohimbine (Y). Neonatal rats were evaluated after $8 \mathrm{~h}$ exposure to $30 \mathrm{mg} /$ $\mathrm{kg}$ propofol (P2 group), post-injection of 1, 10 or $20 \mu \mathrm{g} / \mathrm{kg}$ DEX (D1, D2 or D3 group), co-exposure to 1, 10, or $20 \mu \mathrm{g} / \mathrm{kg}$ DEX $1 \mathrm{~h}$ before $30 \mathrm{mg} / \mathrm{kg}$ propofol (D1/D2/D3+P2 group), and yohimbine $1 \mu \mathrm{g} / \mathrm{kg} 0.5 \mathrm{~h}$ before the similar injection regimen with DEX and propofol (Y+D1/D2/D3+P2 group), respectively. Data are means \pm SD from three independent experiments. ${ }^{* * *} \mathrm{p}<0.0001$ vs. the control group, ${ }^{\# \# \# *} \mathrm{p}<0.0001$ vs. $\mathrm{P} 2$ group, ${ }^{\&} \mathrm{p}<0.05,{ }^{\text {\&\&\& }} \mathrm{p}<0.001$ vs. $\mathrm{D} 2 / \mathrm{D} 3+\mathrm{P} 2$ group. 

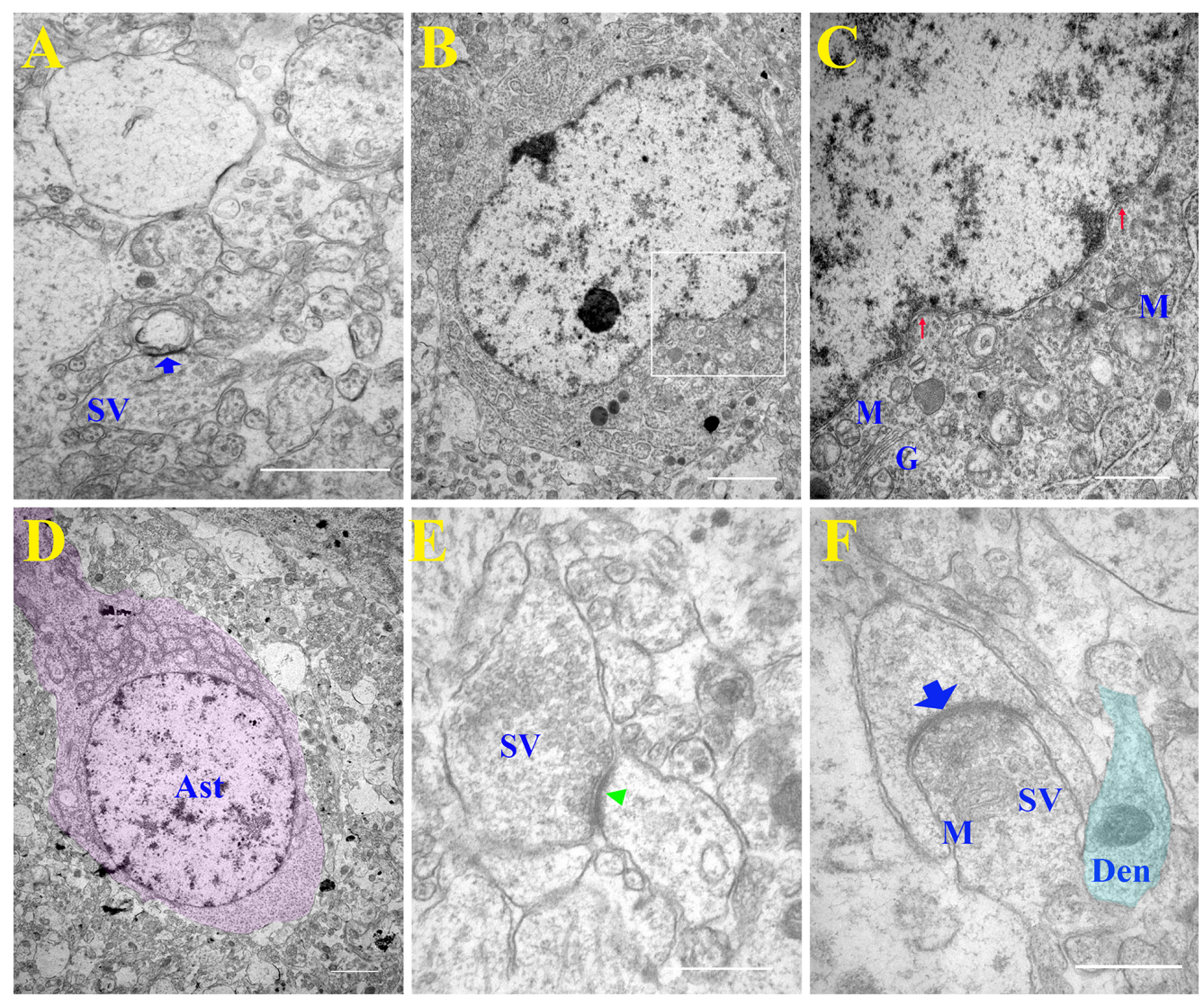

Fig. 6. TEM analysis of the hippocampal cellular and synaptic ultrastructure from P7 rats. (A) Symmetrical synapses (blue arrows) enriched with multiple SVs are visible in the control group. Scale bar $=1 \mu \mathrm{m}$. (B) Nucleus with intact nuclear envelop and increased heterochromatin agglutination is evident in the P2 group (propofol $30 \mathrm{mg} / \mathrm{kg} ; 8 \mathrm{~h}$ ). Details in the rectangle are shown in C. Scale bar $=2 \mu \mathrm{m}$. (C) Local dimpled appearance (red arrows), with obviously diminished organelles. Decreased mitochondrial crista and vacuolation, M. Golgi apparatus, G. (D) In the D1 group (dexmedetomidine, DEX $1 \mu \mathrm{g} /$ $\mathrm{kg}$ ), heterochromatin is well distributed among the nuclei of astrocytes (Ast, light purple), and within the dark-stained filaments in the cytoplasm. Scale bar $=2 \mu \mathrm{m}$. (E, F) In the D2 or D3 group (DEX at the concentration of 10 or $20 \mu \mathrm{g} / \mathrm{kg}$ ), SVs with clearly distinguishable boundaries are abundant. Normal asymmetrical synapses (green arrow heads) and symmetrical synapses (blue arrows) with dense PSDs are visible. Longitudinal section of a dendrite (Den, blue $)$ is highlighted. Mitochondrion, M. Scale bar $=1 \mu \mathrm{m}$. (G) In the D1+P2 group ( $1 \mu \mathrm{g} / \mathrm{kg}$ DEX $1 \mathrm{~h}$ before propofol exposure), the asymmetrical synapse (green arrow heads) contains distinctly diminished numbers of SVs, an obscure synaptic cleft, and an undefined PSD. Scale bar=1 $\mu \mathrm{m}$. (H) In the D2+P2 group (10 $\mu \mathrm{g} / \mathrm{kg}$ DEX $1 \mathrm{~h}$ before propofol exposure), the symmetric synapse (blue arrows) contains a wide cleft and thin PSD. Spine apparatus (sa) inside the dendrite spine. (I) In the D3+P2 group ( $20 \mu \mathrm{g} / \mathrm{kg}$ DEX $1 \mathrm{~h}$ before propofol exposure), multiple tiny glycogen granules (g) are scattered within the cytoplasm. Endoplasmic reticulum (ER) shows slight edema. The synaptic cleft of the asymmetric synapse (green arrow heads) is wide with abundant clear SVs. (J) In the Y+D1+P2 group ( $1 \mu \mathrm{g} / \mathrm{kg}$ yohimbine $0.5 \mathrm{~h}$ before the addition of $1 \mu \mathrm{g} / \mathrm{kg}$ DEX in propofol-exposed rats), three asymmetric synapses (green arrow heads) containing microtubules ( $\mathrm{t}$ ) and SVs surround the axon. Mitochondrial cristae and membrane are broken down. (K) In the Y+D2+P2 group $(1 \mu \mathrm{g} / \mathrm{kg}$ yohimbine $0.5 \mathrm{~h}$ before the addition of $10 \mu \mathrm{g} / \mathrm{kg}$ DEX in propofol-exposed rats), a synapse with a wide cleft (yellow arrow) and thin PSD is visible. Den (light blue) is highlighted. (L) In the Y+D3+P2 group ( $1 \mu \mathrm{g} / \mathrm{kg}$ yohimbine $0.5 \mathrm{~h}$ before the addition of $20 \mu \mathrm{g} / \mathrm{kg}$ DEX in propofol-exposed rats), an obscure membrane in the asymmetric synapse (green arrow heads) can be seen with lower numbers of SVs. Multiple filaments are distributed within the thin astrocyte processes (Asp, light purple). Spine apparatus (sa) inside a Den is visible. Scale bar=500 nm unless stated otherwise ( $\mathrm{n}=3$ ).

swimming path length and swimming speeds were also exhibited and, again, no significant differences in age were seen in the 1- and 2-month-old rats (trace picture and data not shown). Furthermore, a typical trajectory seen for locating the platform during the probe tests is depicted in Fig. 9C and 9F. Although the animals received a single application of anesthesia at the brain peak developmental period, the anesthetic and control rats showed no significant acquisition or memory retention deficits at juvenile age.

\section{DISCUSSION}

Synaptic plasticity, including synaptic structural and functional plasticity, is the modification of synaptic transmission in early neural development. The relationship between them is thought to be multifaceted. In neonates, single propofol exposure affected the synaptic ultrastructure and further suppressed LTP in juvenile rats, independantly. However, we found that the spatial learning 

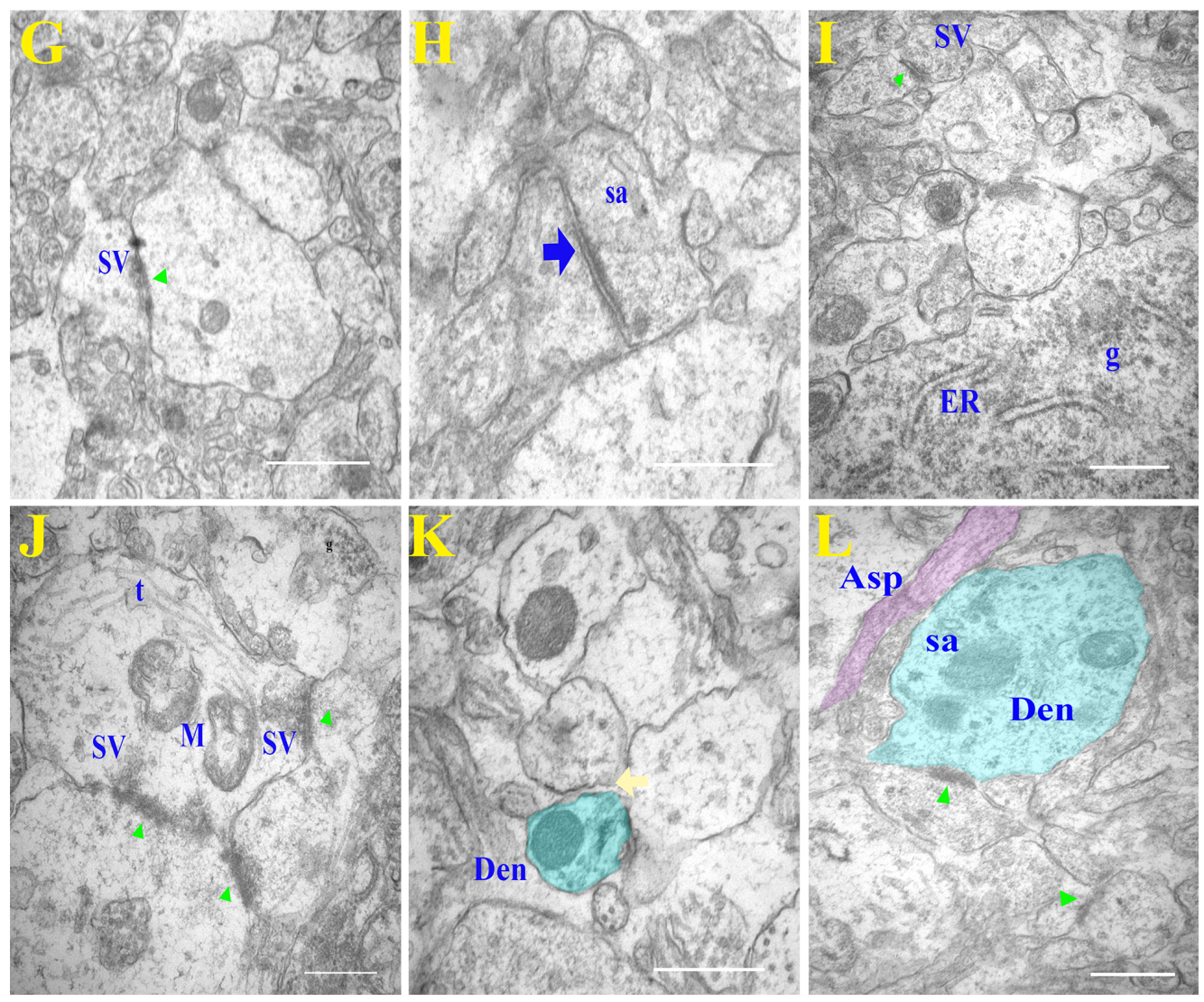

Fig. 6. Continued.

memory during early adolescence (P30) and young adults (P60) in anesthetic treated rats was not significantly different to the control group. The reason may be that a consensus between electrophysiological and memory changes has yet to be reached and there is no absolute cause and effect. This is in agreement with others, where a single injection of propofol $(60 \mathrm{mg} / \mathrm{kg})$, administered at the neonatal peak period of CNS development, was insufficient to cause cognitive dysfunction of juvenile mice [10]. However, Xiao et al [11] found that 9 week old rats showed aberrant neurological deficits after receiving the $100 \mathrm{mg} / \mathrm{kg}$ propofol (50 mg/ $/ \mathrm{kg}$, twice) at the neonatal period. The discrepant reason may be due to their different drug regimen, which somehow decreased number of neurocytes, self-renewal and differentiation capacity of neural stem cells during the period of mature brains. On the one hand, it is acknowledged that the anesthetic mechanism of propofol in the developing CNS relates to multiple biological changes, including inhibition of network connections, inhibition of synaptic transmission efficiency and depression of neuronal activity [12]. On the other hand, the Schaffer collateral CAl pathway is not the only pathway involved in LTP, thus there may be additional connections that could represent an alternative mechanism for long- term cognition alleviation. That is to say, the neurological basic of learning, memory, and behavior is not solely associated with specific synaptic circuits. With self-renewal and maturation, the detrimental influence on synaptogenesis induced by propofol during the growth spurt period of the mammalian neural system, which peaks at P7 in rodents [13], is gradually ameliorated in mature brains.

Programmed cell death is a fundamental process involved in the maintenance of stable neuronal networks. It has been found that nearly $1 \%$ of all neurons undergo natural apoptosis during the early phase of development [14]. During embryogenesis, the critical step of neural tube development is thought to represent an apoptotic process [15]. During the peak period of rapid synaptogenesis, apoptosis also plays a critical role in synaptic formation and remodeling [16]. Therefore, it is reasonable to assume that any aberration in this process would probably result in damage to cells and cellular destruction, causing neural system malfunction and inhibition of synaptogenesis. Here we studied the changes in apoptosis related genes, including MMP-9, which is one of the most abundant extracellular matrix metalloproteases found in the CNS. MMP-9 has mainly been found in neuroglial cells of 


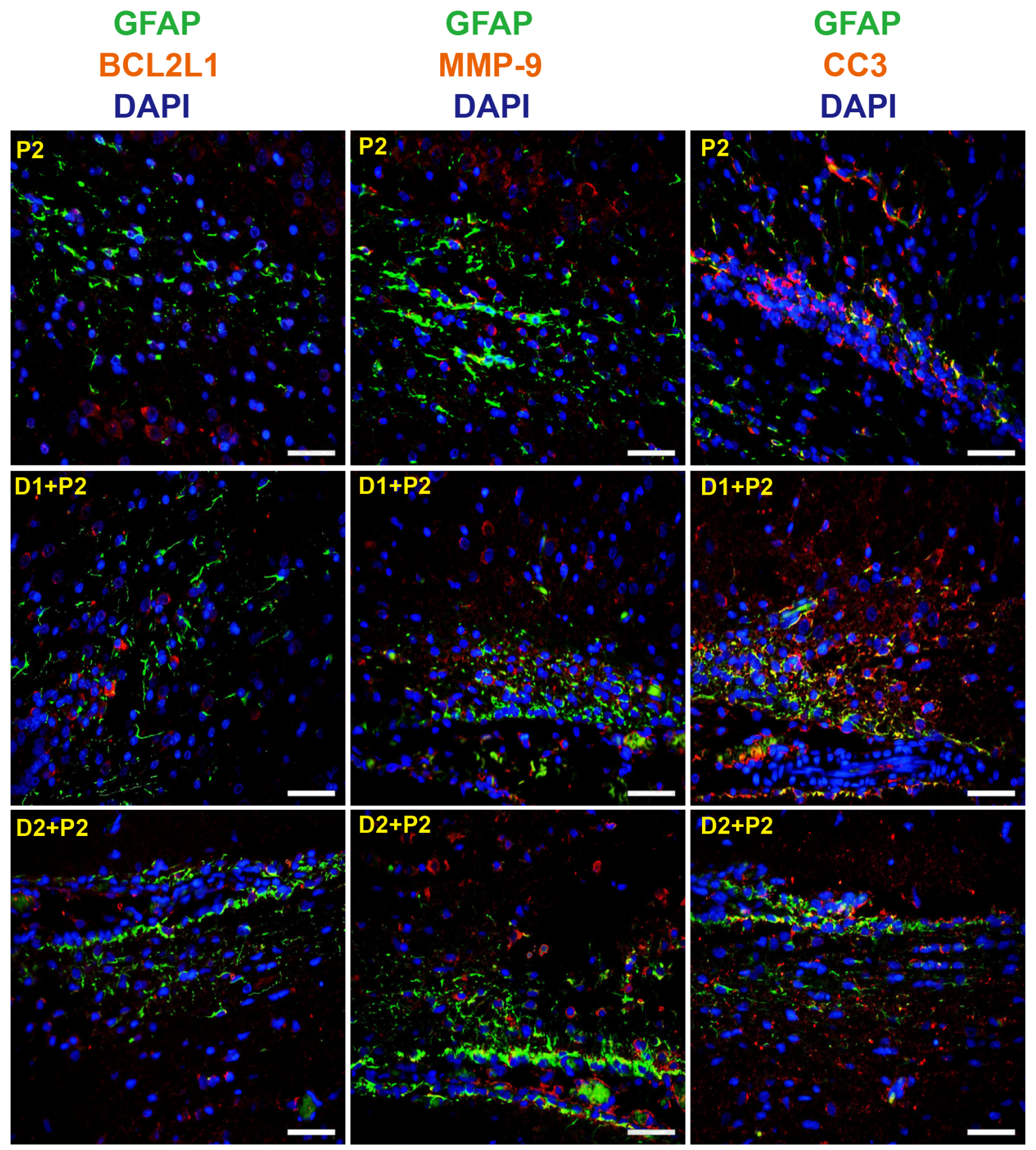

Fig. 7. Double fluorescent micrographs of apoptosis-related genes after exposure to different agents in vivo. The representative double immunofluorescent micrographs and quantitative analysis of the co-localization of GFAP (green) and DAPI (blue) and BCL2L1, MMP-9 and CC3 (all red), as shown in sections representing $8 \mathrm{~h}$ exposure to propofol (P2 group), co-exposure to 1, 10, or $20 \mu \mathrm{g} / \mathrm{kg}$ dexmedetomidine (DEX) and propofol (D1/D2/D3+P2 group), or yohimbine and DEX and propofol (Y+D1/D2/D3+P2 group). Scale bar=50 $\mu \mathrm{m}$. Data are means \pm SD from three independent experiments. ${ }_{* * * *} \mathrm{p}<0.0001$ vs. the control group, ${ }^{*} \mathrm{p}<0.05,{ }^{\# *} \mathrm{p}<0.01,{ }^{* \# \# *} \mathrm{p}<0.0001$ vs. P2 group, ${ }^{\& \&} \mathrm{p}<0.01,{ }^{\& \& \&} \mathrm{p}<0.001,{ }^{\text {\&\&\& } \&} \mathrm{p}<0.0001$ vs. D1/D2/D3+P2 group. 


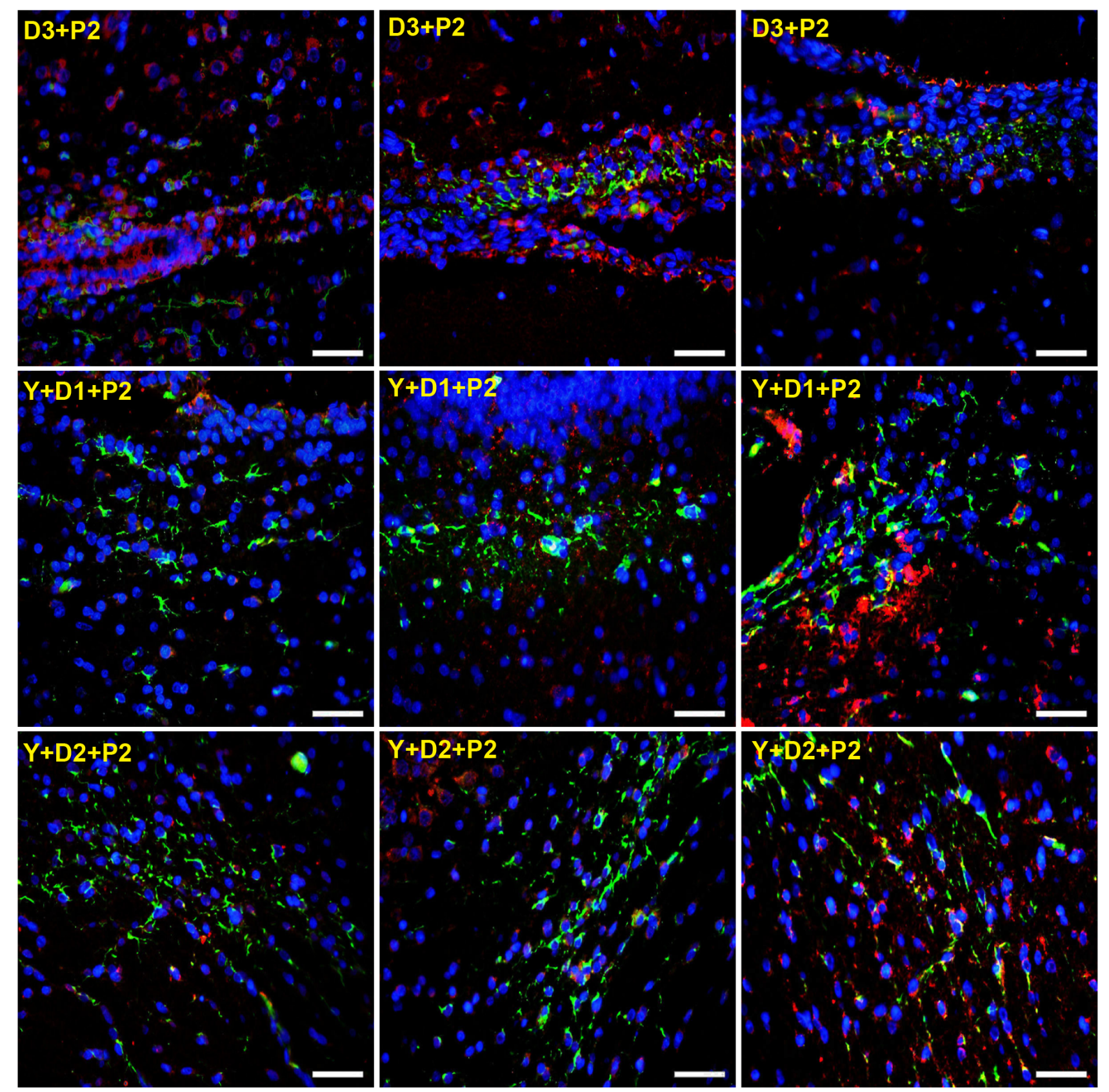

Fig. 7. Continued.

the hippocampus, cortex and cerebellum [10], and has extremely high expression levels and activity in the developing mammalian brain [17]. Therefore, it is conceivable that the levels of MMP-9 may decrease when neonatal hippocampal neurocytes underwent apoptosis after a single injection of propofol [18]. Moreover, it has been suggested that the apoptotic insults would depress MMP9 expression, which would cause an inhibition in the level of the anti-apoptotic gene Bcl-xl. Propofol decreased the level of MMP9, which is the molecular basis of maintaining LTP, suggesting that propofol appears to be neurotoxic to the memory consolidation process by down regulating MMP-9 [19]. In the CNS, MMP-9 selectively targets the physiology of the synapse and behavioral plasticity, but its specific role in synaptogenesis or maturation in morphology is still unclear [20]. To our knowledge, this is the first study to show that neonatal exposure to propofol inhibits MMP-9 levels following apoptosis of neurocytes in the peak developmental period of the brain.

DEX is a widely applied auxiliary anesthetic suitable for a bal- 

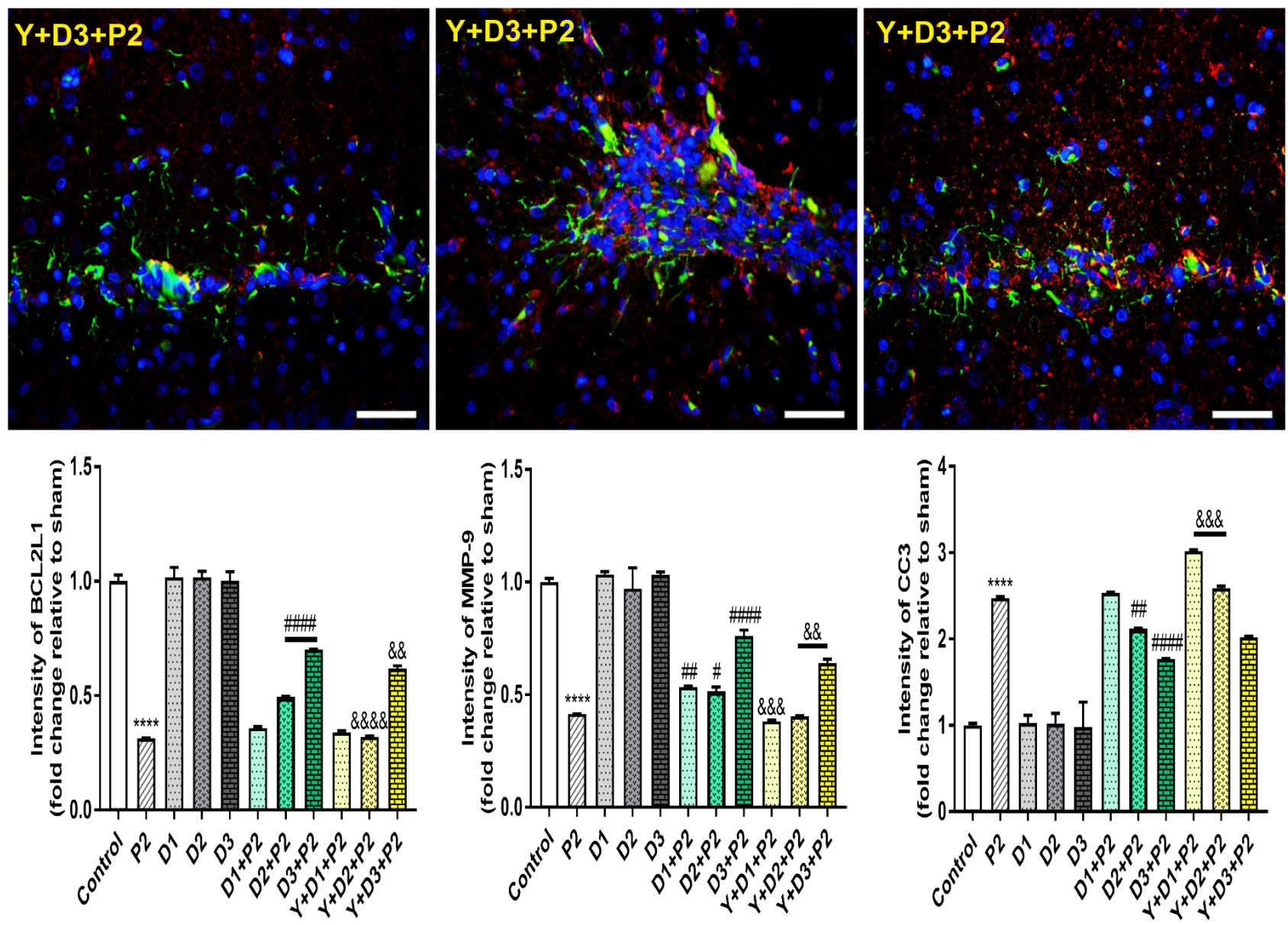

Fig. 7. Continued.

anced anesthetic technique. The antiapoptotic property of DEX is suggested to be involved in the $\mathrm{Bcl}-2$ apoptotic signaling pathway seen in the present study, and the augmentation of the apoptotic effector CC3, in parallel with a reduction in BCL2L1. DEX administration at $1 \mathrm{~h}$ before propofol application also suppressed apoptosis by inhibiting CC3 activation. Even at a dose of $30 \mu \mathrm{g} /$ $\mathrm{kg}$, DEX itself cannot induce apoptosis in fetal cynomolgus monkey brains [21]. However, it must be emphasized that DEX per se does not induce neuronal apoptosis at clinical doses [22-24], which could ameliorate neurocyte apoptosis induced by other common general anesthetics such as propofol. Furthermore, this antiapoptotic effect could be reversed by the presynaptic $\alpha 2$ receptor antagonist, yohimbine, which enhanced propofol neurotoxicity in neonatal rodent hippocampi. When DEX is used at a higher dose, it can cause neurodegeneration because at these doses it has the ability to bind a1-adrenergic receptors $[25,26]$. It firstly revealed that the therapeutic mechanisms of DEX preconditioning rescued the neurotoxicity induced by propofol, such as reducing apoptosis in developing hippocampal neurocytes and impairment of synaptic plasticity. A pretreatment of DEX could effectively reverse the neurotoxicity seen on developing synapses in propofoltreated, immature rats. One of the potential mechanisms of action is the involvement of some neurotransmitters such as GABA and glutamate in the process of neurodevelopment and all common clinically relevant general anesthetics bind to GABA or N-methyl$\mathrm{D}$-aspartic acid receptors. Therefore, if such receptors have been occupied by anesthetics, then their endogenous ligands will not be able to bind, leading to synaptogenesis deficits [14]. However, this explanation may not be relevant to DEX, as it does not interact with the above receptors. Nevertheless, the mechanism of action of DEX as a neuroprotective agent, and its ability to rescue long-term synaptic plasticity, still requires further elucidation.

There are some limitations to our study that require highlighting. First, results are obviously limited to investigations in rodent species and therefore, it is difficult to extrapolate this finding to humans directly. Second, during adolescence and young adulthood, rats showed no aberrant neurological deficits after receiving the single application of the general anesthetic at the neonatal period. 

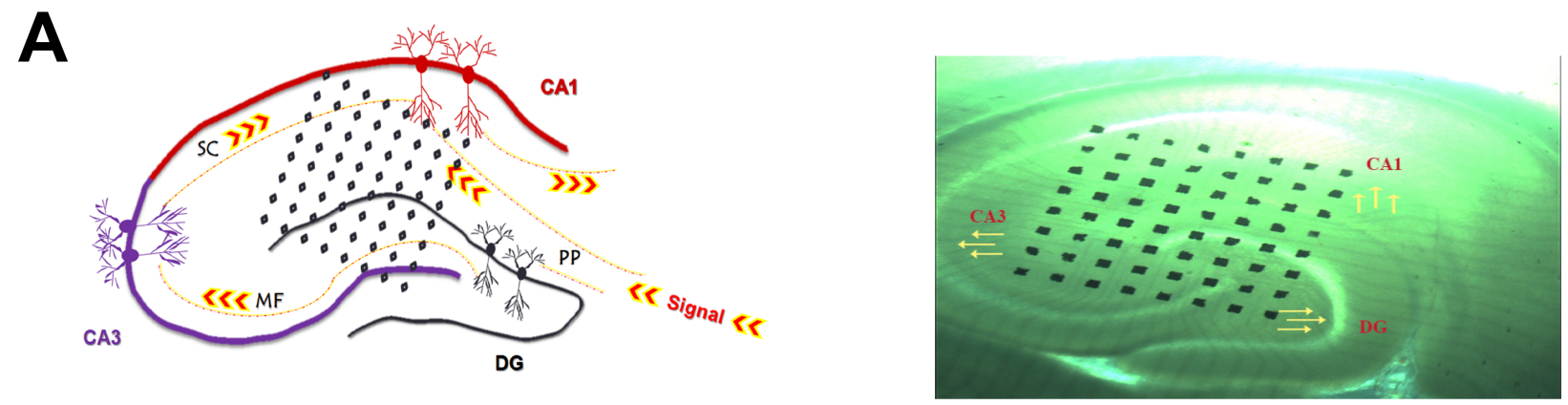

B
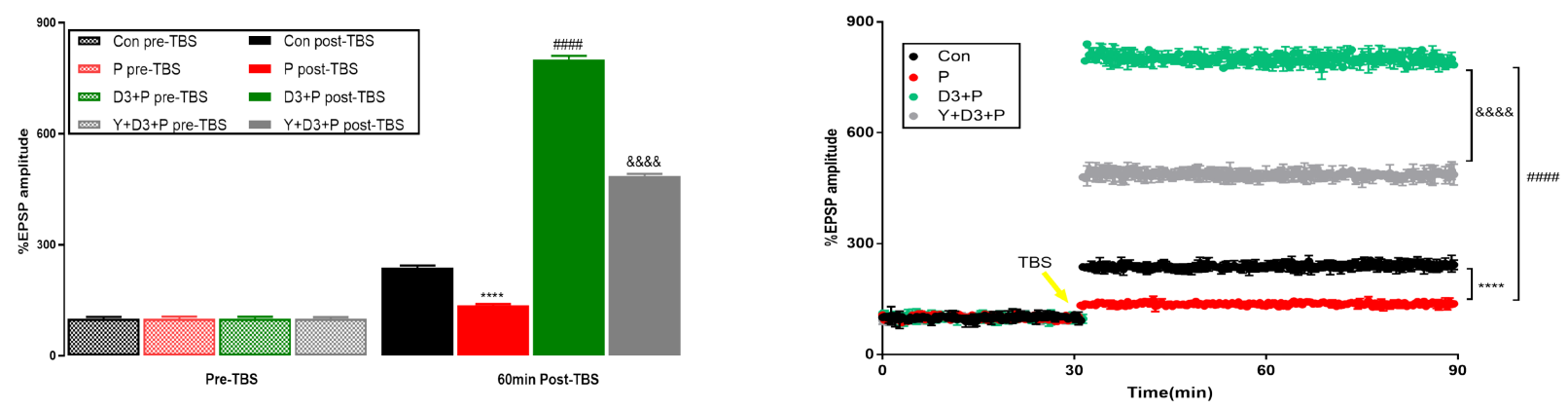

C
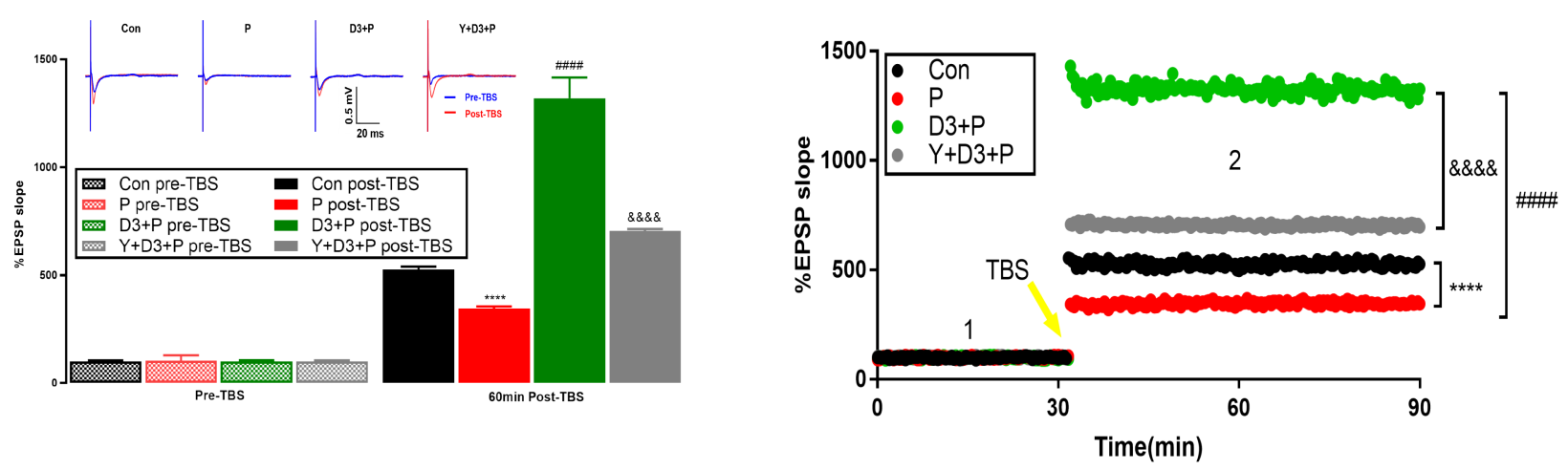

Fig. 8. Effect of a single neonatal propofol exposure on the electrophysiology and apoptotic signaling in P30 rodent hippocampi. (A) Schematic diagram, and photomicrograph of the Med 64 probe position on DG and CA1 regions. DG, dentate gyrus; MF, mossy fiber; PP, perforant pathway; SC, Schaffer collaterals. (B) The fEPSP magnitude in the ex-vivo electrophysiological assay. The long term results at P30 following a single neonatal injection of propofol or $20 \mu \mathrm{g} / \mathrm{kg}$ dexmedetomidine (DEX) was designated as P group or D3 group. After the co-exposure regimen executed on P7, P30 rats were designated as D3+P group ( $20 \mu \mathrm{g} / \mathrm{kg}$ DEX $1 \mathrm{~h}$ before propofol dosing) and Y+D3+P2 group (yohimbine $1 \mu \mathrm{gg} / \mathrm{kg} 0.5 \mathrm{~h}$ before the similar injection regimen with DEX and propofol). Propofol significantly reduced the magnitude of the increase in LTP, indicating a deleterious effect of propofol on synaptic plasticity. DEX restored LTP in acute hippocampal slices from P30 rats. Yohimbine (Y) impaired LTP by inhibiting fEPSP, when compared to DEXtreated rats. (C) Data represent a significant increase in the fEPSP slope. (D) Western blotting of MMP-9, BCL2L1 and CC3 in the hippocampi of P30 rats. $\beta$-actin was used as internal control. (E) mRNA expression of MMP-9, BCL2L1 and CC3 in the hippocampi of P30 rats. ${ }^{* * *} \mathrm{p}<0.0001$ vs. control group, ${ }^{\# \#} \mathrm{p}<0.01,{ }_{\# \# \#} \mathrm{p}<0.0001$ vs. P group, ${ }^{\&} \mathrm{p}<0.05,{ }^{\& \& \&} \mathrm{p}<0.01,{ }^{\text {\&\&\&\& } 8} \mathrm{p}<0.0001$ vs. $\mathrm{D} 3+\mathrm{P}$ group $(\mathrm{n}=3$ animals per group).

But many uncertain factors (e.g., the pre-existing undiagnosed neurological problems, lack of surgical stress, untreated pain, and different paradigm of anesthetics) which accelerate neurodevelopment during late adulthood after neonatal anesthesia exposure were not be fully addressed and therefore, it is premature to preclude the long-term neurotoxic effect of general anesthetics on the immature brains of mammalians. Certainly, more research is needed to reveal the delicate interplay between the various components within neural networks during development.

In summary, we found that DEX preconditioning alleviated the change in expression levels of MMP-9/BCL2L1/CC3 in apoptotic signaling inhibited by a single neonatal injection of propofol. Not only did this produce attenuation of apoptotic neurodegeneration caused by apoptosis, but it also normalized the propofol-induced 
D

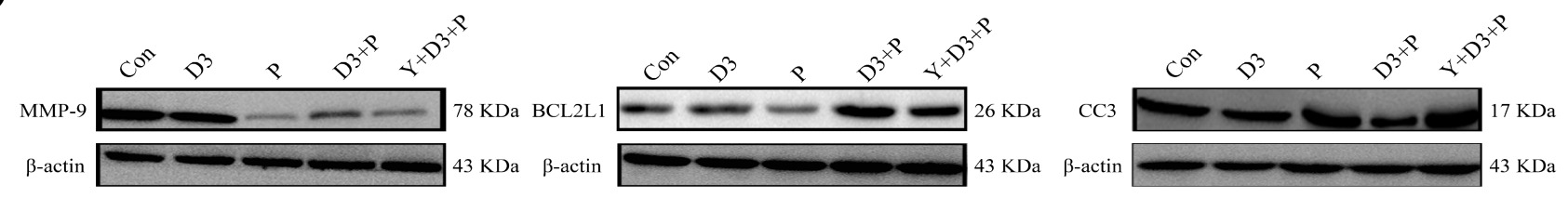

- Control - D3 A P $\circ$ D3+P $\nabla$ Y+D3+P
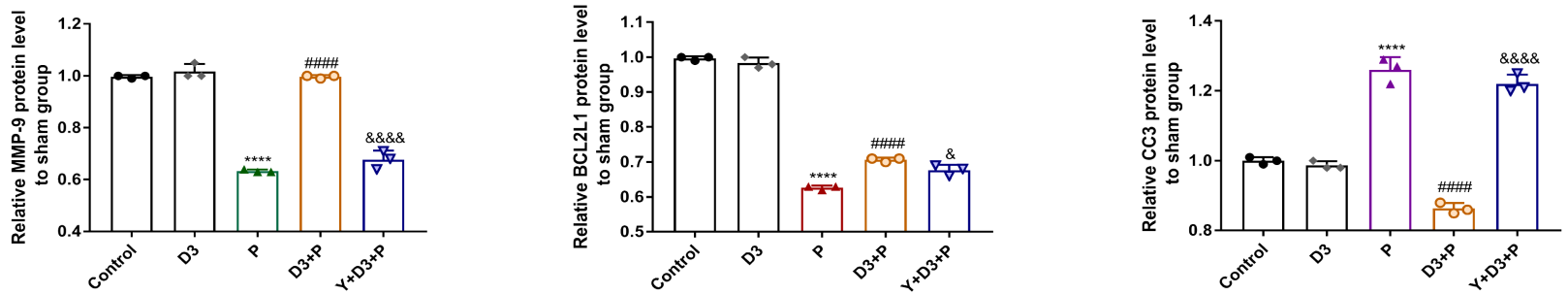

E
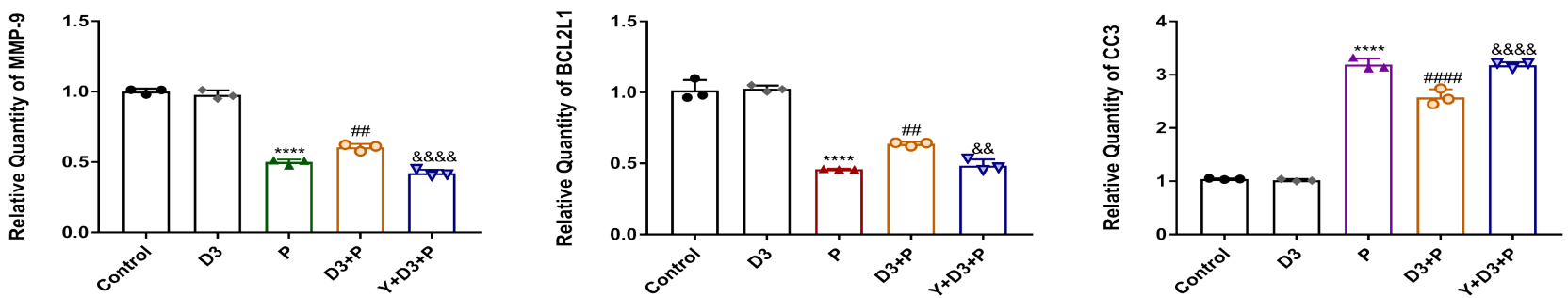

Fig. 8. Continued.

aberrant synaptic plasticity in juvenile rodent hippocampi. Although a single exposure to propofol in neonates induced no longterm dysfunction of spatial discrimination or memory that persisted into adolescence, DEX pretreatment protected the integrity of the developing hippocampal neurocytes and synaptic structures against propofol neurotoxicity.

\section{ACKNOWLEDGEMENTS}

This work was supported by the National Natural Science Foundation of China (No. 81701121).

\section{REFERENCES}

1. Backeljauw B, Holland SK, Altaye M, Loepke AW (2015) Cognition and brain structure following early childhood surgery with anesthesia. Pediatrics 136:e1-e12.

2. Liang X, Zhang Y, Zhang C, Tang C, Wang Y, Ren J, Chen X, Zhang Y, Zhu Z (2017) Effect of repeated neonatal sevoflurane exposure on the learning, memory and synaptic plasticity at juvenile and adult age. Am J Transl Res 9:4974-4983.

3. Osman ES, Khafagy HF, Samhan YM, Hassan MM, ElShanawany FM, Fathallah AR, El-Fandy GG (2012) In vivo effects of different anesthetic agents on apoptosis. Korean J Anesthesiol 63:18-24.

4. Yu D, Jiang Y, Gao J, Liu B, Chen P (2013) Repeated exposure to propofol potentiates neuroapoptosis and long-term behavioral deficits in neonatal rats. Neurosci Lett 534:41-46.

5. Liu F, Rainosek SW, Sadovova N, Fogle CM, Patterson TA, Hanig JP, Paule MG, Slikker W Jr, Wang C (2014) Protective effect of acetyl-L-carnitine on propofol-induced toxicity in embryonic neural stem cells. Neurotoxicology 42:49-57.

6. Ma D, Hossain M, Rajakumaraswamy N, Arshad M, Sanders RD, Franks NP, Maze M (2004) Dexmedetomidine produces its neuroprotective effect via the alpha $2 \mathrm{~A}$-adrenoceptor subtype. Eur J Pharmacol 502:87-97.

7. Moser MB, Moser EI, Forrest E, Andersen P, Morris RG (1995) Spatial learning with a minislab in the dorsal hippocampus. Proc Natl Acad Sci U S A 92:9697-9701.

8. Morris R (1984) Developments of a water-maze procedure for studying spatial learning in the rat. J Neurosci Methods 11:47-60.

9. Hussaini SMQ, Jang MH (2018) New roles for old glue: astrocyte function in synaptic plasticity and neurological disorders. Int Neurourol J 22(Suppl 3):S106-S114.

10. Stawarski M, Stefaniuk M, Wlodarczyk J (2014) Matrix me- 

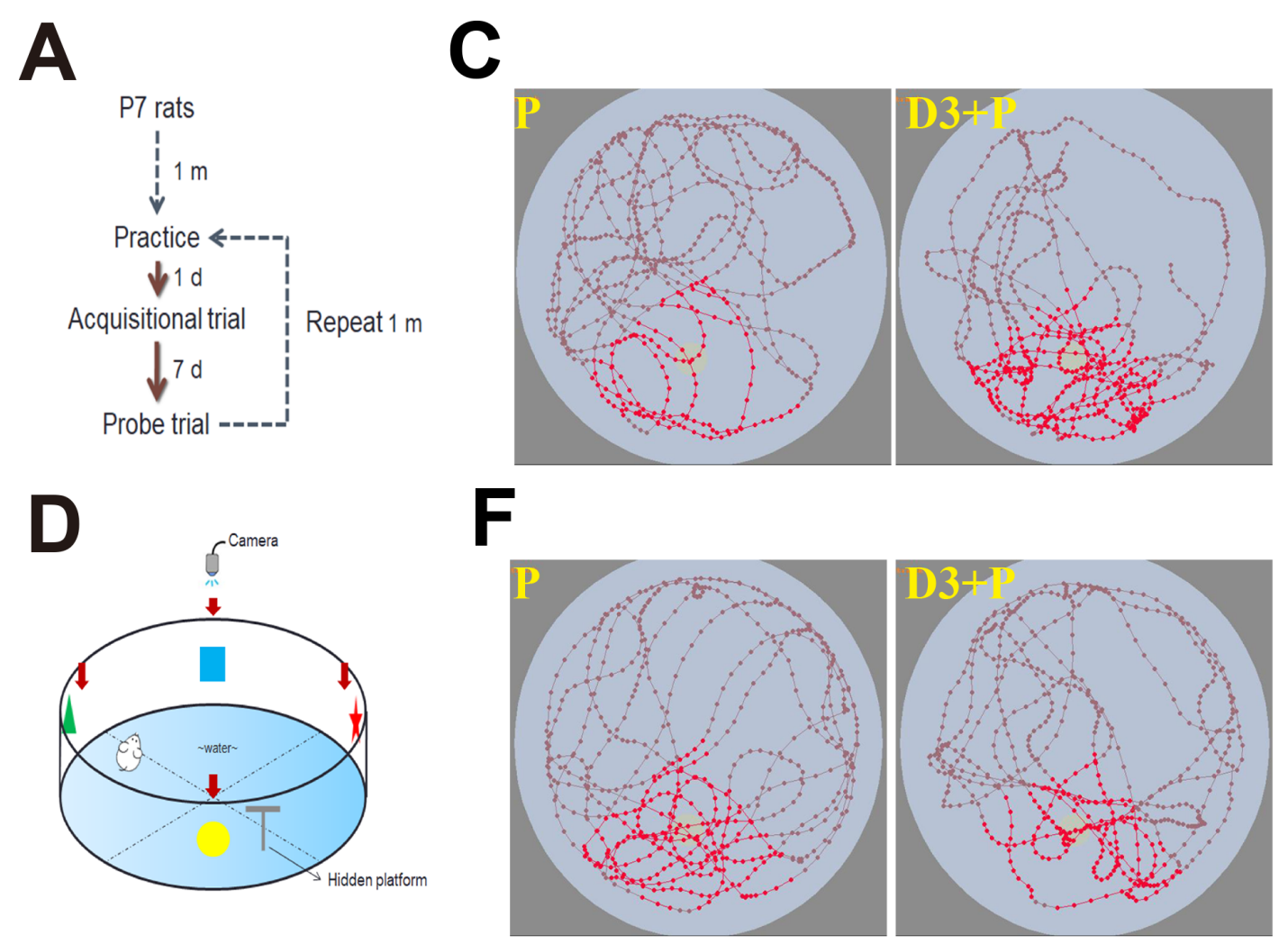

Fig. 9. Absence of long-lasting cognitive dysfunction after a single neonatal propofol infusion. (A) The workflow diagram of water maze test. (B) During the acquisition phase, no significant difference in escape latency to locate the submerged platform (Quadrant 3 is the target quadrant) was seen. The probe task, data of target quadrant crossing times, platform crossing times, and latency to first crossing platform showed no significant difference among all groups at P30 (p>0.05). (C) Representative swim trace from different groups was captured after removing the platform on the probe day (incomplete display). Swimming path taken by anesthetic-exposed rats at age 1 month was no longer than controls in the probe test. Circles filled in light yellow symbolize the location of the removed platform in quadrant III. Red dashed lines show the route the swimming rats took in the target quadrant and the brown dashed lines represent the rat location in the trial. (D) Experimental design. (E) In each trial index on 2-month-old rats, there were no significant differences seen during the acquisition or probe phases ( $p>0.05$ ). (F) Swim trace pictures of 2-month-old rats showed no cognition impairment among the groups (incomplete display) ( $\mathrm{n}=3$ animals per group). The long term results at P30 and P60 were both manifested as P group (30 mg/kg propofol), D1/D2/D3 group (1, 10 or $20 \mu \mathrm{g} / \mathrm{kg}$ DEX alone injection), D1/D2/D3+P group (co-exposure to 1, 10, or $20 \mu \mathrm{g} / \mathrm{kg}$ DEX $1 \mathrm{~h} \mathrm{before} 30 \mathrm{mg} / \mathrm{kg}$ propofol), and $\mathrm{Y}+\mathrm{D} 1 / \mathrm{D} 2 / \mathrm{D} 3+\mathrm{P}$ group $(1 \mu \mathrm{g} / \mathrm{kg}$ yohimbine $0.5 \mathrm{~h}$ before the similar injection regimen with DEX and propofol $)$, seperately. All drugs were intraperitoneally injected on P7.

talloproteinase- 9 involvement in the structural plasticity of dendritic spines. Front Neuroanat 8:68.

11. Xiao Y, Zhou L, Tu Y, Li Y, Liang Y, Zhang X, Lv J, Zhong Y, Xie $Y$ (2018) Dexmedetomidine attenuates the propofol-induced long-term neurotoxicity in the developing brain of rats by enhancing the PI3K/Akt signaling pathway. Neuropsychiatr Dis Treat 14:2191-2206.

12. Lunardi N, Oklopcic A, Prillaman M, Erisir A, JevtovicTodorovic V (2015) Early exposure to general anesthesia disrupts spatial organization of presynaptic vesicles in nerve terminals of the developing rat subiculum. Mol Neurobiol 52:942-951.

13. Dobbing J, Sands J (1979) Comparative aspects of the brain growth spurt. Early Hum Dev 3:79-83.
14. Andropoulos DB (2018) Effect of anesthesia on the developing brain: infant and fetus. Fetal Diagn Ther 43:1-11.

15. Chi H, Sarkisian MR, Rakic P, Flavell RA (2005) Loss of mitogen-activated protein kinase kinase kinase 4 (MEKK4) results in enhanced apoptosis and defective neural tube development. Proc Natl Acad Sci U S A 102:3846-3851.

16. Rice D, Barone S Jr (2000) Critical periods of vulnerability for the developing nervous system: evidence from humans and animal models. Environ Health Perspect 108 Suppl 3(Suppl 3):511-533.

17. Ethell IM, Ethell DW (2007) Matrix metalloproteinases in brain development and remodeling: synaptic functions and targets. J Neurosci Res 85:2813-2823.

18. Kamat PK, Swarnkar S, Rai S, Kumar V, Tyagi N (2014) Astro- 
B
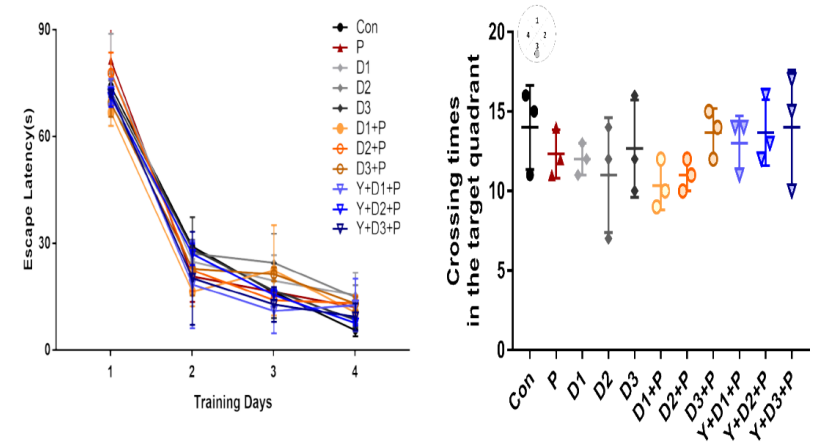

E
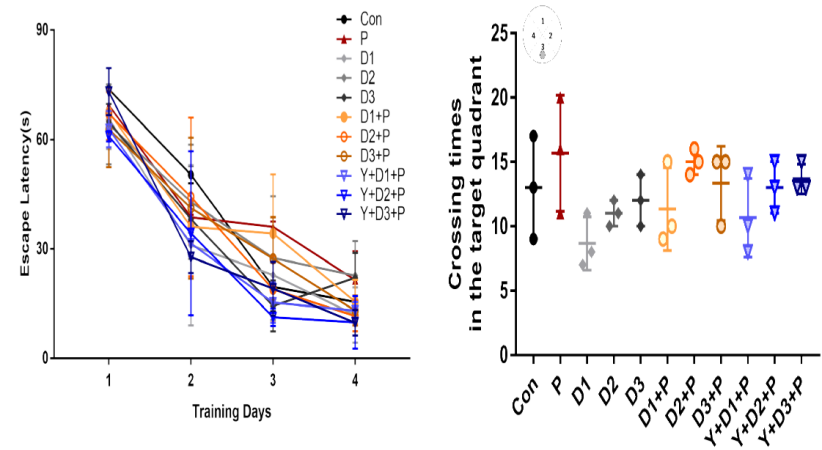
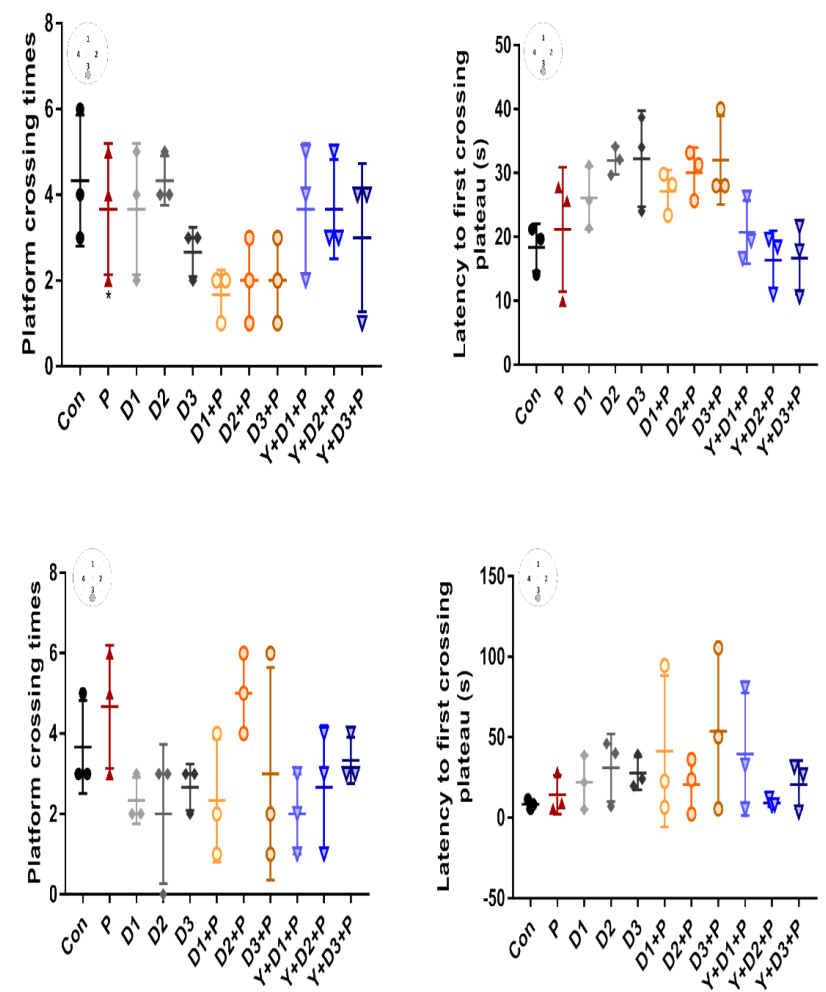

Fig. 9. Continued.

cyte mediated MMP-9 activation in the synapse dysfunction: an implication in Alzheimer disease. Ther Targets Neurol Dis $1: \mathrm{e} 243$.

19. Zhang J, Zhang X, Jiang W (2013) Propofol impairs spatial memory consolidation and prevents learning-induced increase in hippocampal matrix metalloproteinase- 9 levels in rat. Neuroreport 24:831-836.

20. Nagy V, Bozdagi O, Matynia A, Balcerzyk M, Okulski P, Dzwonek J, Costa RM, Silva AJ, Kaczmarek L, Huntley GW (2006) Matrix metalloproteinase-9 is required for hippocampal late-phase long-term potentiation and memory. J Neurosci 26:1923-1934.

21. Koo E, Oshodi T, Meschter C, Ebrahimnejad A, Dong G (2014) Neurotoxic effects of dexmedetomidine in fetal cynomolgus monkey brains. J Toxicol Sci 39:251-262.

22. Sanders RD, Sun P, Patel S, Li M, Maze M, Ma D (2010) Dexmedetomidine provides cortical neuroprotection: impact on anaesthetic-induced neuroapoptosis in the rat developing brain. Acta Anaesthesiol Scand 54:710-716.

23. Li J, Xiong M, Nadavaluru PR, Zuo W, Ye JH, Eloy JD, Bekker A (2016) Dexmedetomidine attenuates neurotoxicity induced by prenatal propofol exposure. J Neurosurg Anesthesiol 28:51-64.

24. Sanders RD, Xu J, Shu Y, Januszewski A, Halder S, Fidalgo A, Sun P, Hossain M, Ma D, Maze M (2009) Dexmedetomidine attenuates isoflurane-induced neurocognitive impairment in neonatal rats. Anesthesiology 110:1077-1085.

25. Pancaro C, Segal BS, Sikes RW, Almeer Z, Schumann R, Azocar RJ, Marchand JE (2016) Dexmedetomidine and ketamine show distinct patterns of cell degeneration and apoptosis in the developing rat neonatal brain. J Matern Fetal Neonatal Med 29:3827-3833.

26. Liu JR, Yuki K, Baek C, Han XH, Soriano SG (2016) Dexmedetomidine-induced neuroapoptosis is dependent on its cumulative dose. Anesth Analg 123:1008-1017. 